\title{
Closed expressions for averages of set partition statistics
}

\author{
Bobbie Chern ${ }^{1}$, Persi Diaconis ${ }^{2}$, Daniel M Kane ${ }^{3}$ and Robert C Rhoades ${ }^{4 *}$
}

*Correspondence:

rob.rhoades@gmail.com

${ }^{4}$ Center for Communications

Research, 805 Bunn Dr., Princeton, NJ 08540, USA

Full list of author information is

available at the end of the article

\begin{abstract}
In studying the enumerative theory of super characters of the group of upper triangular matrices over a finite field, we found that the moments (mean, variance, and higher moments) of novel statistics on set partitions of $[n]=\{1,2, \cdots, n\}$ have simple closed expressions as linear combinations of shifted bell numbers. It is shown here that families of other statistics have similar moments. The coefficients in the linear combinations are polynomials in $n$. This allows exact enumeration of the moments for small $n$ to determine exact formulae for all $n$.
\end{abstract}

\section{Background}

The set partitions of $[n]=\{1,2, \cdots, n\}$ (denoted $\Pi(n)$ ) are a classical object of combinatorics. In studying the character theory of upper triangular matrices (see section 'Set partitions, enumerative group theory, and super characters' for background) we were led to some unusual statistics on set partitions. For a set partition $\lambda$ of $n$, consider the dimension exponent (Table 1).

$$
d(\lambda):=\sum_{i=1}^{\ell}\left(M_{i}-m_{i}+1\right)-n
$$

where $\lambda$ has $\ell$ blocks, $M_{i}$ and $m_{i}$ are the largest and smallest elements of the $i$ th block. How does $d(\lambda)$ vary with $\lambda$ ? As shown below, its mean and second moment are determined in terms of the Bell numbers $B_{n}$

$$
\begin{aligned}
\sum_{\lambda \in \Pi(n)} d(\lambda) & =-2 B_{n+2}+(n+4) B_{n+1} \\
\sum_{\lambda \in \Pi(n)} d^{2}(\lambda) & =4 B_{n+4}-(4 n+15) B_{n+3}+\left(n^{2}+8 n+9\right) B_{n+2}-(4 n+3) B_{n+1}+n B_{n} .
\end{aligned}
$$

The right hand sides of these formulae are linear combinations of Bell numbers with polynomial coefficients. Dividing by $B_{n}$ and using asymptotics for Bell numbers (see section 'Asymptotic analysis') in terms of $\alpha_{n}$, the positive real solution of $u e^{u}=n+1$ (so $\left.\alpha_{n}=\log (n)-\log \log (n)+\cdots\right)$ gives

$$
\begin{aligned}
E(d(\lambda)) & =\left(\frac{\alpha_{n}-2}{\alpha_{n}^{2}}\right) n^{2}+O\left(\frac{n}{\alpha_{n}}\right) \\
\operatorname{VAR}(d(\lambda)) & =\left(\frac{\alpha_{n}^{2}-7 \alpha_{n}+17}{\alpha_{n}^{3}\left(\alpha_{n}+1\right)}\right)^{2} n^{3}+O\left(\frac{n^{2}}{\alpha_{n}}\right) .
\end{aligned}
$$

(C) 2014 Chern et al.; licensee Springer. This is an Open Access article distributed under the terms of the Creative Commons Attribution License (http://creativecommons.org/licenses/by/4.0), which permits unrestricted use, distribution, and reproduction in any medium, provided the original work is properly credited. 
Table $1 \mathrm{~A}$ table of the dimension exponent $f(n, 0, d)$

\begin{tabular}{lrrrrrrrrrrrrr}
\hline $\boldsymbol{n} \backslash \boldsymbol{d}$ & $\mathbf{0}$ & $\mathbf{1}$ & $\mathbf{2}$ & $\mathbf{3}$ & $\mathbf{4}$ & $\mathbf{5}$ & $\mathbf{6}$ & $\mathbf{7}$ & $\mathbf{8}$ & $\mathbf{9}$ & $\mathbf{1 0}$ & $\mathbf{1 1}$ & $\mathbf{1 2}$ \\
\hline 0 & 1 & & & & & & & & & & & \\
1 & 1 & & & & & & & & & & & \\
2 & 2 & & & & & & & & & & & \\
3 & 4 & 1 & & & & & & & & & & \\
4 & 8 & 4 & 3 & & & & & & & & & \\
5 & 16 & 12 & 13 & 9 & 2 & & & & & & & \\
6 & 32 & 32 & 42 & 42 & 35 & 12 & 8 & & & & & \\
7 & 64 & 80 & 120 & 145 & 159 & 133 & 86 & 52 & 32 & 6 & & \\
8 & 128 & 192 & 320 & 440 & 559 & 600 & 591 & 440 & 380 & 248 & 164 & 48 & 30 \\
\hline
\end{tabular}

This paper gives a large family of statistics that admit similar formulae for all moments. These include classical statistics such as the number of blocks and number of blocks of size $i$. It also includes many novel statistics such as $d(\lambda)$ and $c_{k}(\lambda)$, the number of $k$ crossings. The number of two crossings appears as the intertwining exponent of super characters.

Careful definitions and statements of our main results are in section 'Statement of the main results'. Section 'Set partitions, enumerative group theory, and super characters' reviews the enumerative and probabilistic theory of set partitions, finite groups, and super characters. Section 'Computational results' gives computational results; determining the coefficients in shifted Bell expressions involves summing over all set partitions for small $n$. For some statistics, a fast new algorithm speeds things up. Proofs of the main theorems are in sections 'Proofs of recursions, asymptotics, and Theorem 3' and 'Proofs of Theorems 1 and 2'. Section 'More data' gives a collection of examples - moments of order up to six for $d(\lambda)$ and further numerical data. In a companion paper [1], the asymptotic limiting normality of $d(\lambda), c_{2}(\lambda)$, and some other statistics is shown.

\section{Statement of the main results}

Let $\Pi(n)$ be the set partitions of $[n]=\{1,2, \cdots, n\}$ (so $|\Pi(n)|=B_{n}$, the $n$th Bell number). A variety of codings are described in section 'Set partitions, enumerative group theory, and super characters'. In this section, $\lambda \in \Pi(n)$ is described as $\lambda=\mathbf{B}_{1}\left|\mathbf{B}_{2}\right| \cdots \mid \mathbf{B}_{\ell}$ with $\mathbf{B}_{i} \cap \mathbf{B}_{j}=\emptyset, \cup_{i=1}^{\ell} \mathbf{B}_{i}=[n]$. Write $i \sim_{\lambda} j$ if $i$ and $j$ are in the same block of $\lambda$. It is notationally convenient to think of each block as being ordered. Let $\operatorname{First}(\lambda)$ be the set of elements of [ $n$ ] which appear first in their block and Last $(\lambda)$ be the set of elements of $[n]$ which occur last in their block. Finally, let $\operatorname{Arc}(\lambda)$ be the set of distinct pairs of integers $(i, j)$ which occur in the same block of $\lambda$ such that $j$ is the smallest element of the block greater than $i$. As usual, $\lambda$ may be pictured as a graph with vertex set $[n]$ and edge $\operatorname{set} \operatorname{Arc}(\lambda)$.

For example, the partition $\lambda=1356|27| 4$, represented in Figure 1, has $\operatorname{First}(\lambda)=$ $\{1,2,4\}, \operatorname{Last}(\lambda)=\{6,7,4\}$, and $\operatorname{Arc}(\lambda)=\{(1,3),(3,5),(5,6),(2,7)\}$.

A statistic on $\lambda$ is defined by counting the number of occurrences of patterns. This requires some notation.

Definition 1. (i) A pattern $\underline{P}$ of length $k$ is defined by a set partition $P$ of $[k]$ and subsets $\mathbf{F}(\underline{P}), \mathbf{L}(\underline{P}) \subset[k]$, and $\mathbf{A}(\underline{P}), \mathbf{C}(\underline{P}) \subset\{[k] \times[k]: i<j\}$. Let $\underline{P}=(P, \mathbf{F}, \mathbf{L}, \mathbf{A}, \mathbf{C})$. 


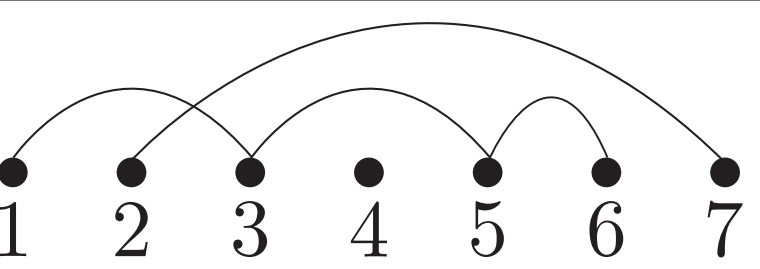

Figure 1 An example partition $\lambda=1356|27| 4$.

(ii) An occurrence of a pattern $\underline{P}$ of length $k$ in $\lambda \in \Pi(n)$ is $s=\left(x_{1}, \cdots, x_{k}\right)$ with $x_{i} \in[n]$ such that

1. $x_{1}<x_{2}<\cdots<x_{k}$.

2. $x_{i} \sim_{\lambda} x_{j}$ if and only if $i \sim_{P} j$.

3. $x_{i} \in \operatorname{First}(\lambda)$ if $i \in \mathbf{F}(\underline{P})$.

4. $x_{i} \in \mathbf{L a s t}(\lambda)$ if $i \in \mathbf{L}(\underline{P})$.

5. $\left(x_{i}, x_{j}\right) \in \operatorname{Arc}(\lambda)$ if $(i, j) \in \mathbf{A}(\underline{P})$.

6. $x_{j}-x_{i}=1$ if $(i, j) \in \mathbf{C}(\underline{P})$.

Write $s \in_{\underline{P}} \lambda$ if $s$ is an occurrence of $\underline{P}$ in $\lambda$.

(iii) A simple statistic is defined by a pattern $\underline{P}$ of length $k$ and $Q \in \mathbb{Z}\left[y_{1}, \cdots, y_{k}, m\right]$. If $\lambda \in \Pi(n)$ and $s=\left(x_{1}, \cdots, x_{k}\right) \in_{\underline{P}} \lambda$, write $Q(s)=\left.Q\right|_{y_{i}=x_{i}, m=n}$. Let

$$
f(\lambda)=f_{\underline{P}, Q}(\lambda):=\sum_{s \in \underline{p} \lambda} Q(s) .
$$

Let the degree of a simple statistic $f_{\underline{P}, Q}$ be the sum of the length of $\underline{P}$ and the degree of $Q$.

(iv) A statistic is a finite $\mathbb{Q}$-linear combination of simple statistics. The degree of a statistic is defined to be the minimum over such representations of the maximum degree of any appearing simple statistic.

Remark 1. In the notation above, $\mathbf{F}(\underline{P})$ is the set of firsts elements, $\mathbf{L}(\underline{P})$ is the set of lasts, A is the arc set of the pattern, and $\mathbf{C}(\underline{P})$ is the set of consecutive elements.

\section{Examples}

1. Number of blocks in $\lambda$ :

$$
\ell(\lambda)=\sum_{\substack{1 \leq x \leq n \\ x \text { is smallest element in its block }}} 1 .
$$

Here, $\underline{P}$ is a pattern of length $1, \mathbf{F}(\underline{P})=\{1\}, \mathbf{L}(\underline{P})=\mathbf{A}(\underline{P})=\mathbf{C}(\underline{P})=\emptyset$, and $Q(y, m)=1$. Similarly, the $n$th moment of $\ell(\lambda)$ can be computed using

$$
\left(\begin{array}{c}
\ell(\lambda) \\
k
\end{array}\right)=f_{\underline{P}_{k}, 1}(\lambda)
$$

where $\underline{P}_{k}$ is the pattern of length $k$ corresponding to $P$, the partition of $[k]$ into blocks of size 1, with $\mathbf{F}\left(\underline{P}_{k}\right)=\{1,2, \cdots, k\}$, and $\mathbf{L}\left(\underline{P}_{k}\right)=\mathbf{A}\left(\underline{P}_{k}\right)=\mathbf{C}\left(\underline{P}_{k}\right)=\emptyset$. 
2. Number of blocks of size $i$ : define a pattern $\underline{P}_{i}$ of length $i$ by: (1) all elements of $[i]$ are equivalent, (2) $\mathbf{F}\left(\underline{P}_{i}\right)=\{1\},(3) \mathbf{L}\left(\underline{P}_{i}\right)=\{i\},(4) \mathbf{A}\left(\underline{P}_{i}\right)=\{(1,2), \cdots,(i-1, i)\}$, and (5) $\mathbf{C}\left(\underline{P}_{i}\right)=\emptyset$. Then,

$$
X_{i}(\lambda):=f_{\underline{P}_{i}, 1}(\lambda)
$$

is the number of $i$ blocks in $\lambda$ (if $i=1, \mathbf{A}\left(\underline{P}_{1}\right)=\emptyset$ ). Similarly, the moments of the number of blocks of size $i$ is a statistic. See Theorem 1 .

3. $k$ crossings: a $k$ crossing [2] of a $\lambda \in \Pi(n)$ is a sequence of arcs $\left(i_{t}, j_{t}\right)_{1 \leq t \leq k} \in \operatorname{Arc}(\lambda)$ with

$$
i_{1}<i_{2}<\cdots<i_{k}<j_{1}<j_{2}<\cdots<j_{k}
$$

The statistic $\operatorname{cr}_{k}(\lambda)$ which counts the number of $k$ crossings of $\lambda$ can be represented by a pattern $\underline{P}=(P, \mathbf{F}, \mathbf{L}, \mathbf{A}, \mathbf{C})$ of length $2 k$ with $(1) i \sim_{P} k+i$ for $i=1, \cdots, k,(2)$ $\mathbf{F}(\underline{P})=\mathbf{L}(\underline{P})=\emptyset,(3) \mathbf{A}(\underline{P})=\{(1, k+1),(2, k+2), \cdots,(k, 2 k)\}$, and $(4) \mathbf{C}(\underline{P})=\emptyset$. Partitions with $c r_{2}(\lambda)=0$ are in bijection with Dyck paths and so are counted by the Catalan numbers $C_{n}=\frac{1}{n+1}\left(\begin{array}{c}2 n \\ n\end{array}\right)$ (see Stanley's second volume on enumerative combinatorics [3]). Partitions without crossings have proved themselves to be very interesting.

Crossing seems to have been introduced by Krewaras [4]. See Simion's [5] for an extensive survey and Chen et al. [2] and Marberg [6] for more recent appearances of this statistic. The statistic $c r_{2}(\lambda)$ appears as the intersection exponent in section 'Super character theory'.

4. Dimension exponent: the dimension exponent described in the introduction is a linear combination of the number of blocks (a simple statistic of degree 1), the last elements of the blocks (a simple statistic of degree 2), and the first elements of the blocks (a simple statistic of degree 2). Precisely, define $f_{\text {firsts }}(\lambda):=f_{\underline{P}, Q}(\lambda)$ where $\underline{P}$ is the pattern of length 1 , with $\mathbf{F}(\underline{P})=\{1\}, \mathbf{L}(\underline{P})=\mathbf{A}(\underline{P})=\mathbf{C}(\underline{P})=\emptyset$, and $Q(y, m)=y$. Similarly, let $f_{\text {lasts }}(\lambda):=f_{\underline{P}, Q}(\lambda)$ where $\underline{P}$ is the pattern of length 1 , with $\mathbf{L}(\underline{P})=\{1\}, \mathbf{F}(\underline{P})=\mathbf{A}(\underline{P})=\mathbf{C}(\underline{P})=\emptyset$, and $Q(y, m)=y$. Then,

$$
d(\lambda)=f_{\text {lasts }}(\lambda)-f_{\text {firsts }}(\lambda)+\ell(\lambda)-n
$$

5. Levels: the number of levels in $\lambda$, denoted $f_{\text {levels }}(\lambda)$, (see page 383 of [7] or Shattuck [8]) is the number of $i$ such that $i$ and $i+1$ appear in the same block of $\lambda$. We have

$$
f_{\text {levels }}(\lambda)=f_{\underline{P}, Q}(\lambda)
$$

where $\underline{P}$ is a pattern of length 2 with $\mathbf{C}(\underline{P})=\mathbf{A}(\underline{P})=\{(1,2)\}, \mathbf{L}(\underline{P})=\mathbf{F}(\underline{P})=\emptyset$, and $Q=1$.

6. The maximum block size of a partition is not a statistic in this notation.

The set of all statistics on $\cup_{n=0}^{\infty} \Pi(n) \rightarrow \mathbb{Q}$ is a filtered algebra.

Theorem 1. Let $\mathcal{S}$ be the set of all set partition statistics thought of as functions $f: \bigcup_{n} \Pi(n) \rightarrow \mathbb{Q}$. Then $\mathcal{S}$ is closed under the operations of pointwise scaling, addition and 
multiplication. In particular, if $f_{1}, f_{2} \in \mathcal{S}$ and $a \in \mathbb{Q}$ then there exist partition statistics $g_{a}, g_{+}, g_{*}$ so that for all set partitions $\lambda$,

$$
\begin{aligned}
a f_{1}(\lambda) & =g_{a}(\lambda) \\
f_{1}(\lambda)+f_{2}(\lambda) & =g_{+}(\lambda) \\
f_{1}(\lambda) \cdot f_{2}(\lambda) & =g_{*}(\lambda) .
\end{aligned}
$$

Furthermore, $\operatorname{deg}\left(g_{a}\right) \leq \operatorname{deg}\left(f_{1}\right), \operatorname{deg}\left(g_{+}\right) \leq \max \left(\operatorname{deg}\left(f_{1}\right), \operatorname{deg}\left(f_{2}\right)\right)$, and $\operatorname{deg}\left(g_{*}\right) \leq$ $\operatorname{deg}\left(f_{1}\right)+\operatorname{deg}\left(f_{2}\right)$. In particular, $\mathcal{S}$ is a filtered $\mathbb{Q}$-algebra under these operations.

Remark 2. Properties of this algebra remain to be discovered.

Definition 2. A shifted Bell polynomial is any function $R: \mathbb{N} \rightarrow \mathbb{Q}$ that is zero or can be expressed in the form

$$
R(n)=\sum_{I \leq j \leq K} Q_{j}(n) B_{n+j}
$$

where $I, K \in \mathbb{Z}$ and each $Q_{j}(x) \in \mathbb{Q}[x]$ such that $Q_{I}(x) \neq 0$ and $Q_{K}(x) \neq 0$. i.e., it is a finite sum of polynomials multiplied by shifted Bell numbers. Call $K$ the upper shift degree of $R$ and I the lower shift degree of $R$.

Remark 3. The representation of a shifted Bell polynomial is unique. This can be understood by considering the asymptotics of each individual term as $n \rightarrow \infty$.

Our first main theorem shows that the aggregate of a statistic is a shifted Bell polynomial.

Theorem 2. For any statistic, fof degree $N$, there exists a shifted Bell polynomial $R$ such that for all $n \geq 1$

$$
M(f ; n):=\sum_{\lambda \in \Pi(n)} f(\lambda)=R(n) .
$$

Moreover,

1. the upper shift index of $R$ is at most $N$ and the lower shift index is bounded below by $-k$, where $k$ is the length of the pattern associated $f$.

2. the degree of the polynomial coefficient of $B_{n+N-j}$ in $R$ is bounded by $j$ for $j \leq N$ and by $j-1$ for $j>N$.

The following collects the shifted Bell polynomials for the aggregates of the statistics given previously.

\section{Examples}

1. Number of blocks in $\lambda$ :

$$
M(\ell ; n)=B_{n+1}-B_{n} .
$$

This is elementary and is established in Proposition 1. 
2. Number of blocks of size $i$ :

$$
M\left(X_{i} ; n\right)=\left(\begin{array}{l}
n \\
i
\end{array}\right) B_{n-i} .
$$

This is also elementary and is established in Proposition 1.

3. Two crossings: Kasraoui [9] established

$$
M\left(c r_{2} ; n\right)=\frac{1}{4}\left(-5 B_{n+2}+(2 n+9) B_{n+1}+(2 n+1) B_{n}\right) .
$$

4. Dimension exponent:

$$
M(d ; n)=-2 B_{n+2}+(n+4) B_{n+1} .
$$

This is given in Theorem 3 .

5. Levels: Shattuck [8] showed that

$$
M\left(f_{\text {levels }} ; n\right)=\frac{1}{2}\left(B_{n+1}-B_{n}-B_{n-1}\right) .
$$

It is amusing that this implies that $B_{3 n} \equiv B_{3 n+1} \equiv 1(\bmod 2)$ and $B_{3 n+2} \equiv 0$ $(\bmod 2)$ for all $n \geq 0$.

Remark 4. Chapter 8 of Mansour's book [7] and the research papers [9-11] contain many other examples of statistics which have shifted Bell polynomial aggregates. We believe that each of these statistics is covered by our class of statistics.

\section{Set partitions, enumerative group theory, and super characters}

This section presents background and a literature review of set partitions, probabilistic and enumerative group theory, and super character theory for the upper triangular group over a finite field. Some sharpenings of our general theory are given.

\section{Set partitions}

Let $\Pi(n, k)$ denote the set partitions of $n$ labeled objects with $k$ blocks and $\Pi(n)=$ $\cup_{k} \Pi(n, k)$; so $|\Pi(n, k)|=S(n, k)$ is the Stirling number of the second kind and $|\Pi(n)|=$ $B_{n}$ is the $n$th Bell number. The enumerative theory and applications of these basic objects is developed in the studies of Graham et al. [12], Knuth [13], Mansour [7], and Stanley [14]. There are many familiar equivalent codings.

- Equivalence relations on $n$ objects

$$
1|2| 3,12|3,13| 2,1 \mid 23,123
$$

- Binary, strictly upper triangular zero-one matrices with no two ones in the same row or column (equivalently, rook placements on a triangular Ferris board (Riordan [15]).

$$
\left(\begin{array}{lll}
0 & 0 & 0 \\
0 & 0 & 0 \\
0 & 0 & 0
\end{array}\right),\left(\begin{array}{lll}
0 & 1 & 0 \\
0 & 0 & 0 \\
0 & 0 & 0
\end{array}\right),\left(\begin{array}{lll}
0 & 0 & 1 \\
0 & 0 & 0 \\
0 & 0 & 0
\end{array}\right),\left(\begin{array}{lll}
0 & 0 & 0 \\
0 & 0 & 1 \\
0 & 0 & 0
\end{array}\right),\left(\begin{array}{lll}
0 & 1 & 0 \\
0 & 0 & 1 \\
0 & 0 & 0
\end{array}\right)
$$

- Arcs on $n$ points

- Restricted growth sequences $a_{1}, a_{2}, \ldots, a_{n} ; a_{1}=0, a_{j+1} \leq 1+\max \left(a_{1}, \ldots, a_{j}\right)$ for $1 \leq j<n$ (Knuth [13], p. 416)

$$
012,001,010,011,000
$$




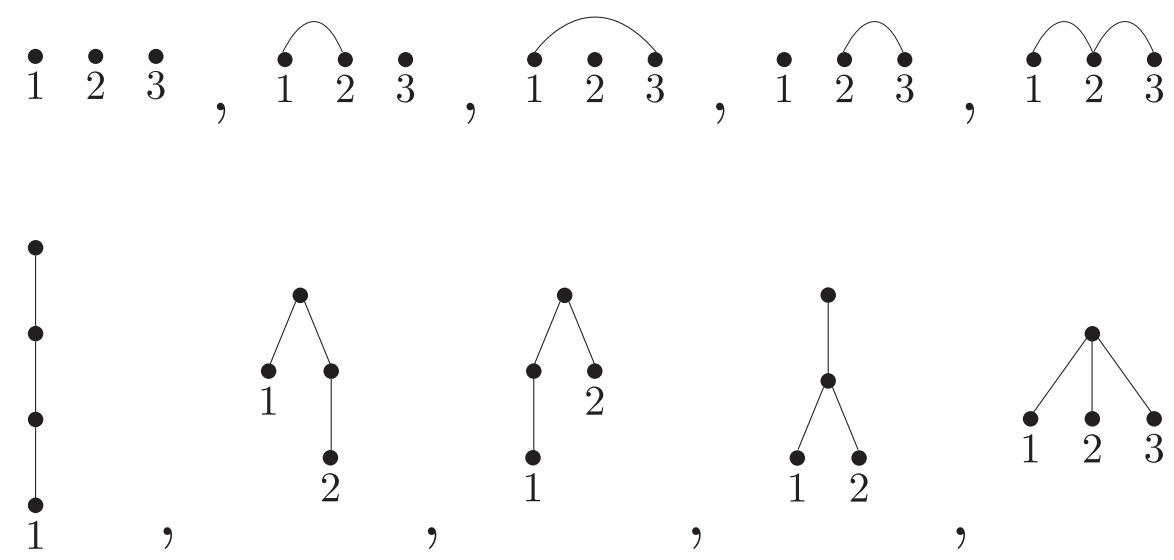

- Semi-labeled trees on $n+1$ vertices

- Vacillating tableau: a sequence of partitions $\lambda^{0}, \lambda^{1}, \ldots, \lambda^{2 n}$ with $\lambda^{0}=\lambda^{2 n}=\emptyset$ and $\lambda^{2 i+1}$ is obtained from $\lambda^{2 i}$ by doing nothing or deleting a square and $\lambda^{2 i}$ is obtained from $\lambda^{2 i-1}$ by doing nothing or adding a square (see [2]).

The enumerative theory of set partitions begins with Bell polynomials. Let $B_{n, k}\left(w_{1}, \cdots, w_{n}\right)=\sum_{\lambda \in \Pi(n, k)} \prod w_{i}^{X_{i}(\lambda)}$ with $X_{i}(\lambda)$ the number of blocks in $\lambda$ of size $i$; so set $B_{n}\left(w_{1}, \cdots, w_{n}\right)=\sum_{k} B_{n, k}\left(w_{1}, \cdots, w_{n}\right)$ and $B(t)=\sum_{n=0}^{\infty} B_{n}(\underline{w}) \frac{t^{n}}{n !}$. A classical version of the exponential formula gives

$$
B(t)=e^{\sum_{n=1}^{\infty} w_{n} \frac{t^{n}}{n !}} .
$$

These elegant formulae have been used by physicists and chemists to understand fragmentation processes ([16] for extensive references). They also underlie the theory of polynomials of binomial type $[17,18]$, that is, families $P_{n}(x)$ of polynomials satisfying

$$
P_{n}(x+y)=\sum P_{k}(x) P_{n-k}(y) .
$$

These unify many combinatorial identities, going back to Faa de Bruno's formula for the Taylor series of the composition of two power series.

There is a healthy algebraic theory of set partitions. The partition algebra of [19] is based on a natural product on $\Pi(n)$ which first arose in diagonalizing the transfer matrix for the Potts model of statistical physics. The set of all set partitions $\bigcup_{n} \Pi(n)$ has a Hopf algebra structure which is a general object of study in [20].

Crossings and nestings of set partitions is an emerging topic, see [2,21,22] and their references. Given $\lambda \in \Pi(n)$ two arcs $\left(i_{1}, j_{1}\right)$ and $\left(i_{2}, j_{2}\right)$ are said to cross if $i_{1}<i_{2}<j_{1}<j_{2}$ and nest if $i_{1}<i_{2}<j_{2}<j_{1}$. Let $\operatorname{cr}(\lambda)$ and $n e(\lambda)$ be the number of crossings and nestings. One striking result: the crossings and nestings are equi-distributed ([21] Corollary 1.5), they show

$$
\sum_{\lambda \in \Pi(n)} x^{c r(\lambda)} y^{n e(\lambda)}=\sum_{\lambda \in \Pi(n)} x^{n e(\lambda)} y^{c r(\lambda)} .
$$

As explained in section 'Super character theory', crossings arise in a group theoretic context and are covered by our main theorem. Nestings are also a statistic. 
This crossing and nesting literature develops a parallel theory for crossings and nestings of perfect matchings (set partitions with all blocks of size 2). Preliminary works suggest that our main theorem carry over to matchings with $B_{n}$ reduced to $(2 n) ! / 2^{n} n !$.

Turn next to the probabilistic side: what does a 'typical' set partition 'look like'? For example, under the uniform distribution on $\Pi(n)$

- What is the expected number of blocks?

- How many singletons (or blocks of size $i$ ) are there?

- What is the size of the largest block?

The Bell polynomials can be used to get moments. For example:

Proposition 1. (i) Let $\ell(\lambda)$ be the number of blocks. Then

$$
\begin{aligned}
& m(\ell ; n):=\sum_{\lambda \in \Pi(n)} \ell(\lambda)=B_{n+1}-B_{n} \\
& m\left(\ell^{2} ; n\right)=B_{n+2}-3 B_{n+1}+B_{n} \\
& m\left(\ell^{3} ; n\right)=B_{n+3}-6 B_{n+2}+8 B_{n+1} B_{n+1}-B_{n}
\end{aligned}
$$

(ii) Let $X_{1}(\lambda)$ be the number of singleton blocks, then

$$
\begin{aligned}
& m\left(X_{1} ; n\right)=n B_{n-1} \\
& m\left(X_{1}^{2} ; n\right)=n B_{n-1}+n(n-1) B_{n-2}
\end{aligned}
$$

In accordance with our general theorem, the right hand sides of (i) and (ii) are shifted Bell polynomials. To make contact with the results shown previously, there is a direct proof of these classical formulae.

Proof. Specializing the variables in the generating function (2) gives a two variable generating functions for $\ell$ :

$$
\sum_{n=0}^{\infty} \sum_{\lambda \in \Pi(n)} y^{\ell(\lambda)} \frac{x^{n}}{n !}=\sum_{\substack{n \geq 0 \\ \ell \geq 0}} S(n, \ell) y^{\ell} \frac{x^{n}}{n !}=e^{y\left(e^{x}-1\right)} .
$$

Differentiating with respect to $y$ and setting $y=1$ shows that $m(\ell ; n)$ is the coefficient of $\frac{x^{n}}{n !}$ in $\left(e^{x}-1\right) e^{e^{x}-1}$. Noting that

$$
\frac{\partial}{\partial x} e^{e^{x}-1}=e^{x} e^{e^{x-1}}=\sum_{n=0}^{\infty} B_{n+1} \frac{x^{n}}{n !}
$$

yields $m(\ell)=B_{n+1}-B_{n}$. Repeated differentiation gives the higher moments.

For $X_{1}$, specializing variables gives

$$
\sum_{n=0}^{\infty} \sum_{\lambda \in \Pi(n)} y^{X_{1}(\lambda)} \frac{x^{n}}{n !}=e^{e^{x}-1-x+y x} .
$$

Differentiation with respect to $y$ and settings $y=1$ readily yields the claimed results.

The moment method may be used to derive limit theorems. An easier, more systematic method is due to Fristedt [23]. He interprets the factorization of the generating function $B(t)$ in (2) as a conditional independence result and uses 'dePoissonization' to get results for finite $n$. Let $X_{i}(\lambda)$ be the number of blocks of size $i$. Roughly, his results say 
that $\left\{X_{i}\right\}_{i=1}^{n}$ are asymptotically independent and of size $(\log (n))^{i} / i$ !. More precisely, let $\alpha_{n}$ satisfy $\alpha_{n} e^{\alpha_{n}}=n+1\left(\operatorname{so} \alpha_{n}=\log (n)-\log \log (n)+o(1)\right)$. Let $\beta_{i}=\alpha_{n}^{i} / i$ ! then

$$
\mathbf{P}\left\{\frac{X_{i}-\beta_{i}}{\sqrt{\beta_{i}}} \leq x\right\}=\Phi(x)+o(1)
$$

where $\Phi(x)=\frac{1}{\sqrt{2 \pi}} \int_{-\infty}^{x} e^{-u^{2} / 2} d u$. Fristedt also has a description of the joint distribution of the largest blocks.

Remark 5. It is typical to expand the asymptotics in terms of $u_{n}$ where $u_{n} e^{u_{n}}=n$. In this notation, $u_{n}$ and $\alpha_{n}$ differ by $O(1 / n)$.

The number of blocks $\ell(\lambda)$ is asymptotically normal when standardized by its mean $\mu_{n} \sim \frac{n}{\log (n)}$ and variance $\sigma_{n}^{2} \sim \frac{n}{\log ^{2}(n)}$. These are precisely given by Proposition 1 . Refining this, Hwang [24] shows

$$
\mathbf{P}\left\{\frac{\ell-\mu_{n}}{\sigma_{n}} \leq x\right\}=\Phi(x)+O\left(\frac{\log (n)}{\sqrt{n}}\right) .
$$

Stam [25] has introduced a clever algorithm for random uniform sampling of set partitions in $\Pi(n)$. He uses this to show that if $W(i)$ is the size of the block containing $i$, $1 \leq i \leq k$, then for $k$ finite and $n$ large $W(i)$ are asymptotically independent and normal with mean and variance asymptotic to $\alpha_{n}$. In [1], we use Stam's algorithm to prove the asymptotic normality of $d(\lambda)$ and $c r_{2}(\lambda)$.

Any of the codings previously mentioned lead to distribution questions. The upper triangular representation leads to the study of the dimension and crossing statistics, the arc representation suggests crossings, nestings, and even the number of arcs, i.e. $n-\ell(\lambda)$. Restricted growth sequences suggest the number of zeros, the number of leading zeros, largest entry. See Mansour [7] for this and much more. Semi-labeled trees suggest the number of leaves, length of the longest path from root to leaf, and various measures of tree shape (e.g., max degree). Further probabilistic aspects of uniform set partitions can be found in $[16,26]$.

\section{Probabilistic group theory}

One way to study a finite group $G$ is to ask what 'typical' elements 'look like'. This program was actively begun by Erdös and Turan [27-33] who focused on the symmetric group $S_{n}$. Pick a permutation $\sigma$ of $n$ at random and ask the following:

- How many cycles in $\sigma$ ? (about $\log n)$

- What is the length of the longest cycle? (about $0.61 n$ )

- How many fixed points in $\sigma$ ? (about 1 )

- What is the order of $\sigma$ ? (roughly $e^{(\log h)^{2} / 2}$ )

In these and many other cases, the questions are answered with much more precise limit theorems. A variety of other classes of groups have been studied. For finite groups of Lie type, see [34] for a survey and [35] for wide-ranging applications. For $p$ groups, see [36].

One can also ask questions about 'typical' representations. For example, fix a conjugacy class $C$ (e.g., transpositions in the symmetric group), what is the distribution of $\chi_{\rho}(C)$ as 
$\rho$ ranges over irreducible representations $[34,37,38]$. Here, two probability distributions are natural, the uniform distribution on $\rho$ and the Plancherel measure $\left(\operatorname{Pr}(\rho)=d_{\rho}^{2} /|G|\right.$ with $d_{\rho}$ the dimension of $\rho$ ). Indeed, the behavior of the 'shape' of a random partition of $n$ under the Plancherel measure for $S_{n}$ is one of the most celebrated results in modern combinatorics. See Stanley's study [39] for a survey with references to the work of Kerov and Vershik [40], Logan and Shepp [41], Baik et al. [42], and many others.

The previous discussion focuses on finite groups. The questions make sense for compact groups. For example, pick a random matrix from Haar measure on the unitary group $U_{n}$ and ask: what is the distribution of its eigenvalues? This leads to the very active subject of random matrix theory. We point to the wonderful monographs of Anderson et al. [43], and Forrester [44] which have extensive surveys.

\section{Super character theory}

Let $G_{n}(q)$ be the group of $n \times n$ matrices which are upper triangular with ones on the diagonal over the field $\mathbb{F}_{q}$. The group $G_{n}(q)$ is the Sylow $p$ subgroup of $\mathrm{GL}_{n}\left(\mathbb{F}_{q}\right)$ for $q=p^{a}$. Describing the irreducible characters of $G_{n}(q)$ is a well-known wild problem. However, certain unions of conjugacy classes, called superclasses, and certain characters, called supercharacters, have an elegant theory. In fact, the theory is rich enough to provide enough understanding of the Fourier analysis on the group to solve certain problems, see the work of Arias-Castro et al. [45]. These superclasses and supercharacters were developed by André [46-48] and Yan [49]. Supercharacter theory is a growing subject. See [6,50-54] and their references.

For the groups $G_{n}(q)$, the supercharacters are determined by a set partition of $[n]$ and a map from the set partition to the group $\mathbb{F}_{q}^{*}$. In the analysis of these characters, there are two important statistics, each of which only depends on the set partition. The dimension exponent is denoted $d(\lambda)$, and the intertwining exponent is denoted $i(\lambda)$.

Indeed, if $\chi_{\lambda}$ and $\chi_{\mu}$ are two supercharacters, then

$$
\operatorname{dim}\left(\chi_{\lambda}\right)=q^{d(\lambda)} \text { and }\left\langle\chi_{\lambda}, \chi_{\mu}\right\rangle=\delta_{\lambda, \mu} q^{i(\lambda)} .
$$

While $d(\lambda)$ and $i(\lambda)$ were originally defined in terms of the upper triangular representation (for example, $d(\lambda)$ is the sum of the horizontal distance from the 'ones' to the super diagonal), their definitions can be given in terms of blocks or arcs:

$$
d(\lambda):=\sum_{e-f \in \operatorname{Arc}(\lambda)}(f-e-1)
$$

and

$$
i(\lambda):=\sum_{\substack{e_{1}<e_{2}<f_{1}<f_{2} \\ e_{1}-f_{1} \in \operatorname{Arc}(\lambda) \\ e_{2}-f_{2} \in \operatorname{Arc}(\lambda)}} 1
$$

Remark 6. Notice that $i(\lambda)=c r_{2}(\lambda)$ is the number of two crossings which were introduced in the previous sections.

Our main theorem shows that there are explicit formulae for every moment of these statistics. The following represents a sharpening using special properties of the dimension exponent. 
Theorem 3. For each $k \in\{0,1,2, \cdots\}$, there exists a closed form expression

$$
M\left(d^{k} ; n\right):=\sum_{\lambda \in \Pi(n)} d(\lambda)^{k}=P_{k, 2 k}(n) B_{n+2 k}+P_{k, 2 k-1}(n) B_{n+2 k-1}+\cdots+P_{k, 0}(n) B_{n}
$$

where each $P_{k, 2 k-j}$ is a polynomial with rational coefficients. Moreover, the degree of $P_{k, 2 k-j}$ is

$$
\left\{\begin{array}{ll}
j & j \leq k \\
k-\left\lceil\frac{j-k}{2}\right\rceil & j>k
\end{array} .\right.
$$

For example,

$$
\begin{aligned}
\sum_{\lambda \in \Pi(n)} d(\lambda) & =-2 B_{n+2}+(n+4) B_{n+1} \\
\sum_{\lambda \in \Pi(n)} d(\lambda)^{2} & =4 B_{n+4}-(4 n+15) B_{n+3}+\left(n^{2}+8 n+9\right) B_{n+2}-(4 n+3) B_{n+1}+n B_{n}
\end{aligned}
$$

Remark 7. See section 'More data' for the moments with $k \leq 6$, and see [55] for the moments with $k \leq 22$. The first moment may be deduced easily from results of Bergeron and Thiem [56]. Note that they seem to have an index which differs by one from ours.

Remark 8 . Theorem 3 is stronger than what is obtained directly from Theorem 2 . For example, the lower shift index is 0 , while the best that can be obtained from Theorem 2 is a lower shift index of $-k$. This theorem is proved by working directly with the generating function for a generalized statistic on 'marked set partitions'. These set partitions are introduced in section 'Computational results'.

Asymptotics for the Bell numbers yield the following asymptotics for the moments. The following result gives some asymptotic information about these moments.

Theorem 4. Let $\alpha_{n}=\log (n)-\log \log (n)+o(1)$ be the positive real solution of $u e^{u}=$ $n+1$. Then

$$
E(d(\lambda))=\left(\frac{\alpha_{n}-2}{\alpha_{n}^{2}}\right) n^{2}+O\left(n \alpha_{n}^{-1}\right) .
$$

Let $S_{k}(d ; n):=\frac{1}{B_{n}} \sum_{\lambda \in \Pi(n)}\left(d(\lambda)-M(d ; n) / B_{n}\right)^{k}$ be the symmetrized moments of the dimension exponent. Then

$$
\begin{aligned}
& S_{2}(d ; n)=\left(\frac{\alpha_{n}^{2}-7 \alpha_{n}+17}{\alpha_{n}^{3}\left(\alpha_{n}+1\right)}\right) n^{3}+O\left(n^{2} \alpha_{n}^{-1}\right) \\
& S_{3}(d ; n)=\left(-\frac{881}{3}-244 \alpha_{n}+145 \alpha_{n}^{2}-\frac{83}{3} \alpha_{n}^{3}+2 \alpha_{n}^{4}\right) \frac{n^{4}}{\alpha_{n}^{4}\left(\alpha_{n}+1\right)^{3}}+O\left(n^{3} \alpha_{n}^{-1}\right)
\end{aligned}
$$

Remark 9. Asymptotics for $S_{k}(d ; n)$ with $k=1,2,3,4,5,6$ and with further accuracy are in section 'More data'.

Analogous to these results for the dimension exponent are the following results for the intertwining exponent. 
Theorem 5. For each $k \in\{0,1,2, \cdots\}$ there exists a closed form expression

$$
M\left(i^{k} ; n\right):=\sum_{\lambda \in \Pi(n)} i(\lambda)^{k}=Q_{k, 2 k}(n) B_{n+2 k}+\cdots+Q_{k, 0}(n) B_{n}+\cdots+Q_{k,-k}(n) B_{n-k}
$$

where each $Q_{k, 2 k-j}$ is a polynomial with rational coefficients. Moreover, the degree of $Q_{k, 2 k-j}$ is bounded by $j$.

For example,

$$
\begin{aligned}
M(i ; n)= & \frac{1}{4}\left((2 n+1) B_{n}+(2 n+9) B_{n+1}-5 B_{n+2}\right) \\
M\left(i^{2} ; n\right)= & \frac{1}{144}\left(\left(36 n^{2}+24 n-23\right) B_{n}+\left(72 n^{2}+72 n-260\right) B_{n+1}\right. \\
& \left.+\left(36 n^{2}+156 n+489\right) B_{n+2}-(180 n+814) B_{n+3}+225 B_{n+4}\right) .
\end{aligned}
$$

Remark 10. The expression for $M(i ; n)=M\left(\mathrm{cr}_{2} ; n\right)$ was established first by Kasraoui (Theorem 2.3 of [9]).

Remark 11. Theorem 5 is deduced directly from Theorem 2. The shifted Bell polynomials for $M\left(i^{k} ; n\right)$ for $k \leq 5$ are given in section 'More data', and see [55] for the aggregates with $k \leq 12$.

Remark 12. Amusingly, the formula for $M(i ; n)$ implies that the sequence $\left\{B_{n}\right\}_{n=0}^{\infty}$ taken modulo 4 is periodic of length 12 beginning with $\{1,1,2,1,3,0,3,1,0,3,3,2\}$. Similarly, the formula for $M\left(i^{2} ; n\right)$ shows that the sequence is periodic modulo 9 (respectively 16) with period 39 (respectively 48). For more about such periodicity, see the papers of Lunnon et al. [57] and Montgomery et al. [58].

In analogy with Theorem 4, there is the following asymptotic result.

Theorem 6. With $\alpha_{n}$ as above,

$$
\begin{aligned}
E(i(\lambda)) & =\left(\frac{2 \alpha_{n}-5}{4 \alpha_{n}^{2}}\right) n^{2}+O\left(n \alpha_{n}^{-1}\right) . \\
\text { Let } S_{k}(i ; n) & =\frac{1}{B_{n}} \sum_{\lambda \in \Pi(n)}\left(i(\lambda)-M(i, n) / B_{n}\right)^{k} . \text { Then, } \\
S_{2}(i ; n) & =\frac{3 \alpha_{n}^{2}-22 \alpha_{n}+56}{9 \alpha_{n}^{3}\left(\alpha_{n}+1\right)} n^{3}+O\left(n^{2} \alpha_{n}^{-1}\right) \\
S_{3}(i ; n) & =\frac{\left(\alpha_{n}-5\right)\left(4 \alpha_{n}^{3}-31 \alpha_{n}^{2}+100 \alpha_{n}+99\right)}{8 \alpha_{n}^{4}\left(\alpha_{n}+1\right)^{3}} n^{4}+O\left(n^{3} \alpha_{n}^{-3}\right)
\end{aligned}
$$

Theorems 3 and 5 show that there will be closed formulae for all of the moments of these statistics. Moreover, these theorems give bounds for the number of terms in the summand and the degree of each of the polynomials. Therefore, to compute the formulae, it is enough to compute enough values for $M\left(d^{k} ; n\right)$ or $M\left(i^{k} ; n\right)$ and then to do linear algebra to solve for the coefficients of the polynomials. For example, $M(d ; n)$ needs $P_{1,2}(n)$ which has degree at most $0, P_{1,1}(n)$ which has degree at most 1 , and $P_{1,0}(n)$ which has degree at most 0 . Hence, there are four unknowns, and so only $M(d ; n)$ for $n=1,2,3,4$ are needed to derive the formula for the expected value of the dimension exponent. 


\section{Computational results}

Enumerating set partitions and calculating these statistics would take time $O\left(B_{n}\right)$ (see Knuth's volume [13] for discussion of how to generate all set partitions of fixed size, the book of Wilf and Nijenhuis [59], or the website [60] of Ruskey). This section introduces a recursion for computing the number of set partitions of $n$ with a given dimension or intertwining exponent in time $O\left(n^{4}\right)$. The recursion follows by introducing a notion of 'marked' set partitions. This generalization seems useful in general when computing statistics which depend on the internal structure of a set partition.

The results may then be used with Theorems 3 and 5 to find exact formulae for the moments. Proofs are given in section 'Proofs of recursions, asymptotics, and Theorem 3'.

For a set partition $\lambda$, mark each block either open or closed. Call such a partition a marked set partition. For each marked set partition $\lambda$ of $[n]$, let $o(\lambda)$ be the number of open blocks of $\lambda$ and $\ell(\lambda)$ be the total number of blocks of $\lambda$. (Marked set partitions may be thought of as what is obtained when considering a set partition of a potentially larger set and restricting it to $[n]$. The open blocks are those that will become larger upon adding more elements of this larger set, while the closed blocks are those that will not.) With this notation, define the dimension of $\lambda$ with blocks $\mathbf{B}_{1}, \mathbf{B}_{2}, \cdots$ by

$$
\tilde{d}(\lambda)=\left(\sum_{\substack{\mathbf{B}_{j} \\ \mathbf{B}_{j} \text { is closed }}} \max \left(\mathbf{B}_{j}\right)\right)-\left(\sum_{\mathbf{B}_{j}} \min \left(\mathbf{B}_{j}\right)\right)+\ell(\lambda)+n(\mathrm{o}(\lambda)-1) .
$$

It is clear that if $o(\lambda)=0$, then $\lambda$ may be thought of as a usual 'unmarked' set partition and $\tilde{d}(\lambda)=d(\lambda)$ is the dimension exponent of $\lambda$. Define

$$
f(n ; A, B):=\{\lambda \in \Pi(n): o(\lambda)=A \text { and } \tilde{d}(\lambda)=B\}
$$

Theorem 7. For $n>0$

$$
\begin{aligned}
f(n ; A, B)= & f(n-1 ; A-1, B-A+1)+f(n-1 ; A, B-A) \\
& +A f(n-1 ; A, B-A+1)+(A+1) f(n-1 ; A+1, B-A) .
\end{aligned}
$$

with initial condition $f(0 ; A, B)=0$ for all $(A, B) \neq(0,0)$ and $f(0 ; 0,0)=1$.

Therefore, to find the number of partitions of $[n]$ with dimension exponent equal to $k$, it suffices to compute $f(n, 0, k)$ for $k$ and $n$. Figure 2 gives the histograms of the dimension exponent when $n=20$ and $n=100$. With increasing $n$, these distributions tend to normal with mean and variance given in Theorem 4 . This approximation is already apparent for $n=20$.

It is not necessary to compute the entire distribution of the dimension index to compute the moment formulae for the dimension exponent. Namely, it is better to implement the following recursion for the moments. 

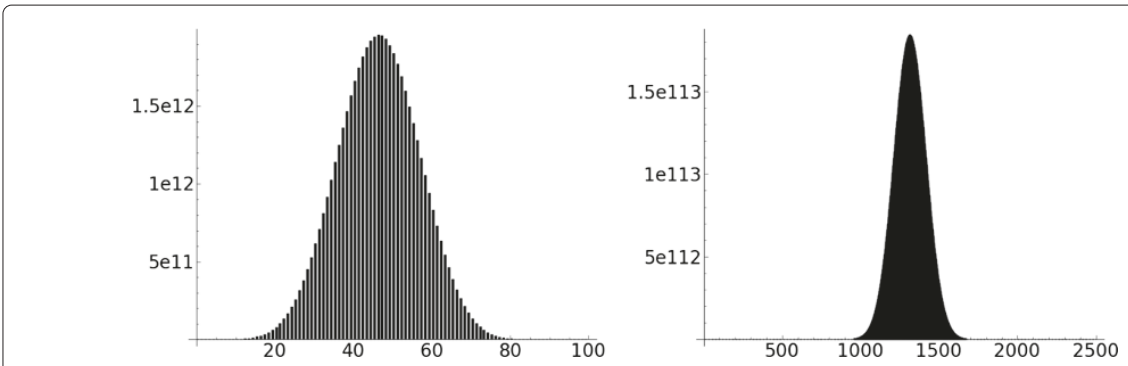

Figure 2 Histograms of the dimension exponent counts for $n=20$ and $n=100$.

Corollary 1. Define $M_{k}(d ; n, A):=\sum_{\substack{\lambda \in \Pi(n) \\ o(\lambda)=A}} d(\lambda)^{k}$. Then

$$
\begin{aligned}
M_{k}(d ; n, A)= & \sum_{j=0}^{k}\left(\begin{array}{l}
k \\
j
\end{array}\right)(A-1)^{k-j} M_{j}(d ; n-1, A-1) \\
& +\sum_{j=0}^{k}\left(\begin{array}{l}
k \\
j
\end{array}\right) A^{k-j} M_{j}(d ; n-1, A) \\
& +A \sum_{j=0}^{k}\left(\begin{array}{l}
k \\
j
\end{array}\right)(A-1)^{k-j} M_{j}(d ; n-1, A) \\
& +(A+1) \sum_{j=0}^{k}\left(\begin{array}{l}
k \\
j
\end{array}\right) A^{k-j} M_{j}(d ; n-1, A+1) .
\end{aligned}
$$

To compute $M\left(d^{k} ; n\right)$, then for each $m<n$, this recursion allows us to keep only $k$ values rather than computing all $O\left(m \cdot m^{2}\right)$ values of $f(m, A, B)$. To find the linear relation of Theorem 3, only $O\left(k \cdot k^{2}\right)$ values of $M_{k}(d ; n, A)$ are needed.

In analogy, there is a recursion for the intertwining exponent.

Let $f_{(i)}(n, A, B)$ be the number of marked partitions of $[n]$ with intertwining weight equal to $B$ and with $A$ open sets where the intertwining weight is equal to the number of interlaced pairs $i \frown j$ and $k \frown \ell$ where $k$ is in a closed set plus the number of triples $i, k, j$ such that $i \frown j$ and $k$ is in an open set.

Theorem 8. With the notation above, the following recursion holds

$$
\begin{aligned}
f_{(i)}(n+1, A, B)= & f_{(i)}(n, A, B)+f_{(i)}(n, A-1, B) \\
& +\sum_{j=0}^{A} f_{(i)}(n, A+1, B-j)+\sum_{j=0}^{A-1} f_{(i)}(n, A, B-j) .
\end{aligned}
$$

This recursion allows the distribution to be computed rapidly. Figure 3 gives the histograms of the intertwining exponent when $n=20$ and $n=100$. Again, for increasing $n$, the distribution tends to normal with mean and variance from Theorem 6 . The skewness is apparent for $n=20$.

\section{Proofs of recursions, asymptotics, and Theorem 3}

This section gives the proofs of the recursive formulae discussed in Theorems 7 and 8 Additionally, this section gives a proof of Theorem 3 using the three-variable generating 

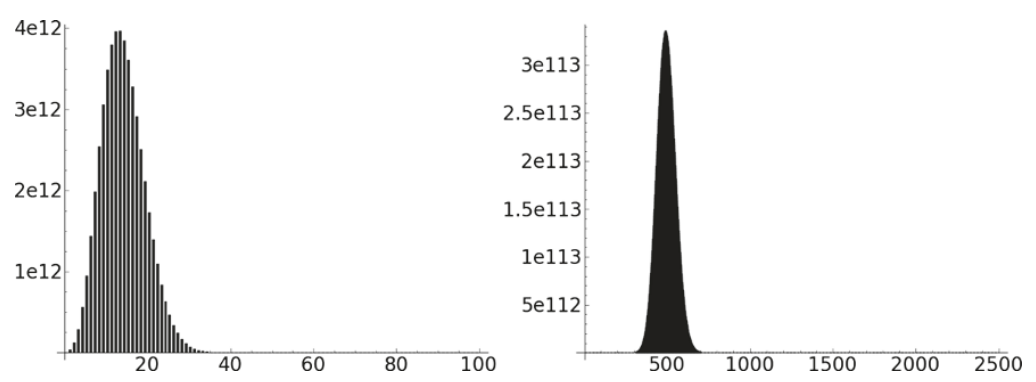

Figure 3 Histograms of the intertwining exponent counts for $n=20$ and $n=100$.

function for $f(n, A, B)$. Finally, it gives an asymptotic expansion for $B_{n+k} / B_{n}$ with $k$ fixed and $n \rightarrow \infty$. This asymptotic is used to deduce Theorems 4 and 6 .

\section{Recursive formulae}

This subsection gives the proof of the recursions for $f(n, A, B)$ and $f_{(i)}(n, A, B)$ given in Theorems 7 and 8 The recursion is used in the next subsection to study the generating function for the dimension exponent.

Proof of Theorem 7 The four terms of the recursion come from considering the following cases: (1) $n$ is added to a marked partition of $[n-1]$ as a singleton open set, (2) $n$ is added to a marked partition of $[n-1]$ as a singleton closed set, (3) $n$ is added to an open set of a marked partition of $[n-1]$ and that set remains open, and (4) $n$ is added to an open set of a marked partition of $[n-1]$ and that set is closed.

Proof of Theorem 8 The argument is similar to that of Theorem 7. The same four cases arise. However, when adding $n$ to an open set, the statistic may increase by any value $j$ and it does so in exactly one way.

\section{The generating function for $f(n, A, B)$}

This section studies the generating function for $f(n, A, B)$ and deduces Theorem 3 . Let

$$
F(X, Y, Z):=\sum_{n, A, B \geq 0} f(n ; A, B) \frac{X^{n}}{n !} Y^{A} Z^{B}
$$

be the three-variable generating function. Theorem 7 implies that

$$
\frac{\partial}{\partial X} F(X, Y, Z)=(1+Y)\left(F(X, Y Z, Z)+F_{Y}(X, Y Z, Z)\right),
$$

where $F_{Y}$ denotes $\frac{\partial}{\partial Y} F$.

Then, $F(X, 0, Z)$ is the generating function for the distribution of $d(\lambda)$, i.e.,

$$
F(X, 0, Z)=\sum_{n=0}^{\infty} \sum_{\lambda \in \Pi(n)} Z^{d(\lambda)} \frac{X^{n}}{n !} .
$$

Thus, the generating function for the $k$ th moment is

$$
\sum_{n \geq 0} M\left(d^{k} ; n\right) \frac{X^{n}}{n !}=\left.\left(Z \frac{\partial}{\partial Z}\right)^{k} F(X, Y, Z)\right|_{Z=1, Y=0} .
$$

Consider

$$
F_{k}(X, Y):=\left.\left(Z \frac{\partial}{\partial Z}\right)^{k} F(X, Y, Z)\right|_{Z=1} .
$$


So $F_{k}(X, 0)=\sum M\left(d^{k} ; n\right) \frac{X^{n}}{n !}$.

Lemma 1. In the notation above,

$$
\left(\frac{\partial}{\partial X}-(1+Y) \frac{\partial}{\partial Y}\right) F_{n}(X, Y)=(1+Y) \sum_{k>0}\left(\begin{array}{l}
n \\
k
\end{array}\right)\left(\left(Y \frac{\partial}{\partial Y}\right)^{k}\left(1+\frac{\partial}{\partial Y}\right) F_{n-k}(X, Y)\right) \text {. }
$$

Proof. From (8),

$$
\frac{\partial}{\partial X} F_{n}(X, Y)=(1+Y) \sum_{k}\left(\begin{array}{l}
n \\
k
\end{array}\right)\left(\left(Y \frac{\partial}{\partial Y}\right)^{k}\left(1+\frac{\partial}{\partial Y}\right) F_{n-k}(X, Y)\right)
$$

Hence, solving for $F_{n}$ gives

$$
\left(\frac{\partial}{\partial X}-(1+Y) \frac{\partial}{\partial Y}\right) F_{n}(X, Y)=(1+Y) \sum_{k>0}\left(\begin{array}{l}
n \\
k
\end{array}\right)\left(\left(Y \frac{\partial}{\partial Y}\right)^{k}\left(1+\frac{\partial}{\partial Y}\right) F_{n-k}(X, Y)\right) .
$$

Throughout the remainder $Y=e^{\alpha}-1$. Abusing notation, let

$$
G_{k}(X, \alpha):=G_{k}(X, Y):=F_{k}(X, Y) \exp \left(-(1+Y)\left(e^{X}-1\right)\right) .
$$

The following lemma gives an expression for $G_{k}(X, \alpha)$ in terms of a differential operators. Define the operators

$$
\begin{aligned}
R & :=\frac{\partial}{\partial X}-\frac{\partial}{\partial \alpha} \\
S & :=e^{\alpha} \\
T & :=\frac{\partial}{\partial \alpha}+e^{X+\alpha} .
\end{aligned}
$$

Lemma 2. Clearly, $G_{0}(X, Y)=1$. Moreover,

$$
G_{k}(X, \alpha)=\sum_{a, b, c} C_{a, b, c}^{k} S^{a} T^{b} X^{c} 1,
$$

Proof. (10) is equivalent to

$$
\begin{aligned}
& \left(\frac{\partial}{\partial X}+(1+Y) e^{X}-(1+Y)\left(\frac{\partial}{\partial Y}+e^{X}\right)\right) G_{n}(X, Y) \\
= & (1+Y) \sum_{k>0}\left(\begin{array}{l}
n \\
k
\end{array}\right)\left(Y\left(\frac{\partial}{\partial Y}+e^{X}-1\right)\right)^{k}\left(\frac{\partial}{\partial Y}+e^{X}\right) G_{n-k}
\end{aligned}
$$

Now

$$
\begin{aligned}
& \left(\frac{\partial}{\partial X}-\frac{\partial}{\partial \alpha}\right) G_{k}(X, \alpha) \\
& \quad=\sum_{\ell>0}\left(\begin{array}{l}
k \\
\ell
\end{array}\right)\left(\left(1-e^{-\alpha}\right)\left(\frac{\partial}{\partial \alpha}+e^{X+\alpha}-e^{\alpha}\right)\right)^{k}\left(\frac{\partial}{\partial \alpha}+e^{X+\alpha}\right) G_{k-\ell}(X, \alpha)
\end{aligned}
$$

where a $e^{\alpha}$ has been commuted through. Then,

$$
R G_{k}(X, \alpha)=\sum_{\ell>0}\left(\begin{array}{l}
k \\
\ell
\end{array}\right)\left(T-T S^{-1}-S\right)^{\ell} T G_{k-\ell} .
$$


Since $G_{k}(0, \alpha)=0$ for $k>0$,

$$
G_{k}(X, \alpha)=\int_{0}^{X} \sum_{\ell>0}\left(\begin{array}{l}
k \\
\ell
\end{array}\right)\left(T-T S^{-1}-S\right)^{\ell} T G_{k-\ell}(t, X+\alpha-t) d t .
$$

From this

$$
G_{k}(X, \alpha)=\sum_{a, b, c} C_{a, b, c}^{k} S^{a} T^{b} X^{c} 1,
$$

for some constants $C_{a, b, c}^{k}$.

The next lemma evaluates the terms in the summation of Lemma 2, thus yielding a generating function for $G_{k}(X, Y)$ which resembles that for the Bell numbers.

\section{Lemma 3.}

$$
\left.\left(T^{\ell} 1\right)\right|_{\alpha=0} \exp \left(e^{X}-1\right)=\sum_{n \geq 0} B_{n+\ell} \frac{X^{n}}{n !} .
$$

Proof. It is easy to see by induction on $\ell$ that $T^{\ell} 1$ is a polynomial in $e^{X+\alpha}$. Thus,

$$
T^{\ell} 1=\left(\frac{\partial}{\partial X}+e^{X+\alpha}\right)^{\ell} 1
$$

Hence

$$
\left.T^{\ell} 1\right|_{\alpha=0}=\left(\frac{\partial}{\partial X}+e^{X}\right)^{\ell} 1
$$

From this, it is easy to see that

$$
\left.T^{\ell} 1\right|_{\alpha=0} \exp \left(e^{X}-1\right)=\frac{\partial^{\ell}}{\partial X^{\ell}} \exp \left(e^{X}-1\right) .
$$

And the result follows.

Lemmas 2 and 3 readily yield the following expression for the moments of the dimension exponent as a shifted Bell polynomial.

Lemma 4. For each $k \geq 0$ and $n \geq 0$

$$
M\left(d^{k} ; n\right)=\sum_{a, b, c} C_{a, b, c}^{k} n(n-1) \cdots(n-c+1) B_{n+b-c} .
$$

Theorem 3 needs some further constraints on the degrees of terms in this polynomial. The following lemma yields the claimed bounds for the degrees.

Lemma 5. In the notation above, $C_{a, b, c}^{k}=0$ unless all of the following hold:

1. $c \leq b$.

2. $c<b$ unless $a=0$.

3. $b \leq 2 k$.

4. $3 c-b \leq k$.

5. $3 c-b \leq k-2$ if $a \neq 0$. 
Proof. Let $H_{a, b, c}(X, \alpha)=S^{a} T^{b} X^{c} 1$. Using Equation 12, write $C_{a, b, c}^{k}$ in terms of the $C_{a, b, c}^{\ell}$ for $\ell<k$. To do this requires understanding

$$
\int_{0}^{X} H_{a, b, c}(t, X+\alpha-t) d t
$$

As a first claim: if $a=0$, then the above is simply $\frac{1}{c+1} H_{0, b, c+1}$. This is seen easily from the fact that $R$ commutes with $T$. For $a \neq 0$, it is easy to see that this is a linear combination of the $H_{a, b, c^{\prime}}$ over $c^{\prime} \leq c$, and of $H_{0, b^{\prime}, 0}$ over $b^{\prime} \leq b$.

The desired properties can now be proved by induction on $k$. It is clear that they all hold for $k=0$. For larger $k$, assume that they hold for all $k-\ell$, and use Equation 12 to prove them for $k$.

By the inductive hypothesis, the $T G_{k-\ell}$ are linear combinations of $H_{a, b, c}$ with $c<b$. Thus, $\left(T-T S^{-1}-S\right)^{\ell} T G_{k-\ell}$ is a linear combination of $H_{a, b, c}$ 's with $b>c$. Thus, by Equation $12, G_{k}$ is a linear combination of $H_{a, b, c}$ 's with $c \leq b$ and $a=0$ or with $c<b$. This proves properties 1 and 2 .

By the inductive hypothesis, the $G_{k-\ell}$ are linear combinations of $H_{a, b, c}$ with $b \leq 2(k-\ell)$. Thus, $T-T S^{-1}-S^{\ell} T G_{k-\ell}$ is a linear combination of $H_{a, b, c}$ 's with $b \leq 2 k+1-\ell \leq 2 k$. Thus, by Equation $12, G_{k}$ is a linear combination of $H_{a, b, c}$ 's with $b \leq 2 k$. This proves property 3.

Finally, consider the contribution to $G_{k}$ coming from each of the $G_{k-\ell}$ terms. For $\ell=1$, $G_{k-\ell}$ is a linear combination of $H_{a, b, c}$ 's with $3 c-b \leq k-3$ if $a \neq 0,3 c-b \leq k-1$ if $a=0$. Thus, $T G_{k-\ell}$ is a linear combination of $H_{a, b, c}$ 's with $3 c-b \leq k-3$ if $a \neq 0$, and $3 c-b \leq k-2$ otherwise. Thus, $\left(T-T S^{-1}-S\right)^{\ell} T G_{k-\ell}$ is a linear combination of $H_{a, b, c}$ 's with $3 c-b \leq k-3$ if $a=0$, and $3 c-b \leq k-2$ otherwise. Thus, the contribution from these terms to $G_{k}$ is a linear combination of $H_{a, b, c}$ 's with $3 c-b \leq k$ and $3 c-b \leq k-2$ if $a \neq 0$. For the terms with $\ell>1, G_{k-\ell}$ is a linear combination of $H_{a, b, c}$ 's with $3 c-b \leq k-2$ and $3 c-b \leq k-4$ when $a \neq 0$. Thus, $T G_{k-\ell}$ is a linear combination of $H_{a, b, c}$ 's with $3 c-b \leq k-3$, as is $\left(T-T S^{-1}-S\right)^{\ell} T G_{k-\ell}$. Thus, the contribution of these terms to $G_{k}$ is a linear combination of $H_{a, b, c}$ 's with $3 c-b \leq k$ and $3 c-b \leq k-3$ if $a \neq 0$. This proves properties 4 and 5 .

This completes the induction and proves the Lemma.

From this Lemma, it is easy to see that

$$
M\left(d^{k} ; n\right)=\sum_{\ell=0}^{2 k} B_{n+\ell} P_{k, \ell}(n)
$$

for some polynomials $P_{k, \ell}(n)$ with $\operatorname{deg}\left(P_{k, \ell}\right) \leq \min (2 k-\ell, k / 2+\ell / 2)$.

\section{Asymptotic analysis}

This section presents some asymptotic analysis of the Bell numbers and ratios of Bell numbers. These results yield Theorems 4 and 6 . Similar analysis can be found in [13].

Proposition 2. Let $\alpha_{n}$ be the solution to

$$
u e^{u}=n+1
$$


and let

$$
\zeta_{n, k}:=e^{\alpha_{n}}\left(1+\frac{1}{\alpha_{n}}\right)+\frac{k}{\alpha_{n}^{2}}=\frac{(n+1)\left(\alpha_{n}+1\right)+k}{\alpha_{n}^{2}} .
$$

Then

$$
B_{n+k}=\frac{(n+k) !}{\sqrt{2 \pi} e} \zeta_{n, k}^{-\frac{1}{2}} \exp \left(e^{\alpha_{n}}-(n+k+1) \log \left(\alpha_{n}\right)\right)\left(1+O\left(e^{-\alpha_{n}}\right)\right) .
$$

More precisely, for $T \geq 0$

$$
\begin{aligned}
B_{n+k}=\frac{(n+k) !}{\sqrt{2 \pi} e} & \zeta_{n, k}^{-\frac{1}{2}} \exp \left(e^{\alpha_{n}}-(n+k+1) \log \left(\alpha_{n}\right)\right) \\
& \times\left(1+\sum_{m=1}^{T} R_{m, k}\left(\alpha_{n}\right) \frac{1}{n^{m}}+O\left(\left(\frac{\alpha_{n}}{n}\right)^{T+1}\right)\right) .
\end{aligned}
$$

where $R_{m, k}$ are rational functions. In particular

$$
\begin{aligned}
R_{1, k}(u)= & \frac{\left(\left(-12 k^{2}+24 k-2\right)+\left(-24 k^{2}+24 k+18\right) u+\left(-12 k^{2}-12 k+20\right) u^{2}+(-12 k+3) u^{3}-2 u^{4}\right)}{24(u+1)^{3}} \\
R_{2, k}(u)= & \frac{\left(1,44 k^{4}-384 k^{3}+624 k^{2}-1,152 k+100\right)+\left(576 k^{4}-576 k^{3}+816 k^{2}-3,264 k-648\right) u}{1152(u+1)^{6}} \\
& +\frac{\left(864 k^{4}+1,056 k^{3}+432 k^{2}-6384 k-1,292\right) u^{2}}{1,152(u+1)^{6}} \\
& +\frac{\left(5,76 k^{4}+2,784 k^{3}+2,280 k^{2}-7,440 k-2,604\right) u^{3}}{1,152(u+1)^{6}} \\
& +\frac{\left(144 k^{4}+2,016 k^{3}+3,888 k^{2}-3,552 k-2,988\right) u^{4}+\left(480 k^{3}+2,328 k^{2}+72 k-1,800\right) u^{5}}{1,152(u+1)^{6}} \\
& +\frac{\left(480 k^{2}+600 k-551\right) u^{6}+(144 k-60) u^{7}+4 u^{8}}{1,152(u+1)^{6}}
\end{aligned}
$$

Proof. The proof is very similar to the traditional saddle point method for approximating $B_{n}$. The idea is to evaluate at the saddle point for $B_{n}$ rather than for $B_{n+k}$. We follow the proof in Chapter 6 of [61].

By Cauchy's formula,

$$
\frac{2 \pi i e}{(n+k) !} B_{n+k}=\int_{C} \exp \left(e^{z}\right) z^{-n-k-1} d z
$$

where $C$ encircles the origin once in the positive direction. Deform the path to a vertical line $u-i \infty$ to $u+i \infty$ by taking a large segment of this line and a large semi-circle going around the origin. As the radius, say $R$, is taken to infinity the factor $z^{-n-k-1}=$ $O\left(R^{-n-k-1}\right)$ and $\exp \left(e^{z}\right)$ is bounded in the half-plane.

Choose $u=\alpha_{n}$ and then

$$
\frac{2 \pi e}{(n+k) !} B_{n+k}=\exp \left(e^{\alpha_{n}}-(n+k+1) \log \left(\alpha_{n}\right)\right) \int_{-\infty}^{\infty} \exp \left(\psi_{n, k}(y)\right) d y
$$

where

$$
\psi_{n, k}(y)=e^{\alpha_{n}}\left(\left(e^{i y}-1\right)-\frac{n+1+k}{e^{\alpha_{n}}} \log \left(1+i y \alpha_{n}^{-1}\right)\right) .
$$

The real part has maxima around $y=2 \pi m$ for each integer $m$, but using log $\left(1+y^{2} \alpha_{n}^{-2}\right)>\frac{1}{2} y^{2} \alpha_{n}^{-2}$ for $\pi<y<\alpha_{n}$ and $1+y^{2} \alpha_{n}^{-2}>2 y \alpha_{n}^{-1}$ for $y>\alpha_{n}$ as in [61] gives

$$
\int_{-\infty}^{\infty} \exp \left(\psi_{n, k}(y)\right) d y=\int_{-\pi}^{\pi} \exp \left(\psi_{n, k}(y)\right) d y+O\left(\exp \left(-\frac{e^{\alpha_{n}}}{\alpha_{n}}\right)\right) .
$$


Next, note that

$$
\begin{aligned}
\psi_{n, k}(y)= & -\frac{i k y}{\alpha_{n}}-\left(1+\frac{n+1+k}{(n+1) \alpha_{n}}\right) \frac{n+1}{\alpha_{n}} \frac{y^{2}}{2} \\
& +\sum_{m>2}\left(\frac{1}{m !}+(-1)^{m} \frac{n+1+k}{m \alpha_{n}^{m-1}(n+1)}\right) \frac{n+1}{\alpha_{n}}(i y)^{m}
\end{aligned}
$$

where $\frac{n+1+k}{e^{\alpha_{n}}}=\alpha_{n}+k e^{-\alpha_{n}}$ and $e^{\alpha_{n}}=\frac{n+1}{\alpha_{n}}$ were used. Hence,

$$
\begin{aligned}
\psi_{n, k}\left(\frac{y}{\sqrt{\zeta_{n, k}}}\right)= & -\frac{i k}{\alpha_{n} \sqrt{\zeta_{n, k}}}-\frac{y^{2}}{2} \\
& +\sum_{m>2}\left(\frac{1}{m !}+(-1)^{m} \frac{n+1+k}{m \alpha_{n}^{m-1}(n+1)}\right) \frac{n+1}{\alpha_{n}}\left(\frac{i y}{\sqrt{\zeta_{n, k}}}\right)^{m}
\end{aligned}
$$

Making the change of variables and extending the sum of interval of integration gives

$$
\begin{aligned}
\int_{-\infty}^{\infty} & \exp \left(\psi_{n, k}(y)\right) d y+O\left(\exp \left(-\frac{e^{\alpha_{n}}}{\alpha_{n}}\right)\right) \\
\quad & =\int_{-\infty}^{\infty} e^{-\frac{y^{2}}{2}} \exp \left(-\frac{i k}{\alpha_{n} \sqrt{\zeta_{n, k}}}+\sum_{m>2} \operatorname{frac} 1 m !+(-1)^{m} \frac{n+1+k}{\alpha_{n}^{m-1}(n+1)} \frac{n+1}{\alpha_{n}}{\frac{i y}{\sqrt{\zeta_{n, k}}}}^{m}\right) d y .
\end{aligned}
$$

Hence, Taylor expanding around $y=0$ and using

$$
\int_{\mathbb{R}} y^{k} e^{-\frac{y^{2}}{2}} d y=\left\{\begin{array}{lll}
0 & k \equiv 1 & (\bmod 2) \\
\sqrt{2 \pi} \frac{k !}{2^{\frac{k}{2}}\left(\frac{k}{2}\right) !} & k \equiv 0 & (\bmod 2)
\end{array}\right.
$$

gives the desired result.

For more details, see [61].

Proposition 2 yields

$$
\frac{B_{n+k}}{B_{n}}=\frac{(n+k) !}{n !} \alpha_{n}^{-k}\left(1-\frac{k \alpha_{n}}{(n+1)\left(\alpha_{n}+1\right)}\right)^{-\frac{1}{2}}\left(1+O\left(e^{-\alpha_{n}}\right)\right) .
$$

Direct application of this result gives the results in Theorems 4 and 6 .

\section{Proofs of Theorems 1 and 2}

This section gives the proofs of Theorems 2 and 1 . Theorem 2 implies Theorem 5 . A pair of lemmas which will be useful in the proof of Theorem 2:

Lemma 6. For $B_{n}$, the Bell numbers, define

$$
g_{r, d, k, s}(n):=n^{d} \sum_{i=0}^{n-k}\left(\begin{array}{c}
n-k \\
i
\end{array}\right) B_{i+s} r^{n-k-i}
$$

where $r, d, k, s$ are non-negative integers. Then, $g_{r, d, k, s}(n)$ is a shifted Bell polynomial of lower shift index $-k$ and upper shift index $r+s-k$.

Proof. It clearly suffices to prove that $g_{r, 0, k, s}(n)$ is a shifted Bell polynomial. Since $g_{r, 0,0, s}(n-k)=g_{r, 0, k, s}(n)$, it suffices to prove that $g_{r, s}(n):=g_{r, 0,0, s}(n)$ is a shifted Bell polynomial. 
For this, consider the exponential generating function

$$
\begin{aligned}
\sum_{n=0}^{\infty} g_{r, s}(n) \frac{x^{n}}{n !} & =\sum_{n=0}^{\infty} \sum_{i=0}^{n}\left(\begin{array}{c}
n \\
i
\end{array}\right) B_{i+s} r^{n-i} \frac{x^{n}}{n !}=\sum_{a=0}^{\infty} \sum_{b=0}^{\infty} B_{a+s} r^{b} \frac{x^{a+b}}{a ! b !} \\
& =\frac{\partial^{s}}{\partial x^{s}}\left(\sum_{n=0}^{\infty} B_{n} \frac{x^{n}}{n !}\right) e^{r x}=e^{r x} \frac{\partial^{s}}{\partial x^{s}}\left(e^{e^{x}-1}\right) .
\end{aligned}
$$

This is easily seen to be equal to $e^{e^{x}-1}$ times a polynomial in $e^{x}$ of degree $s+r$.

Since $g_{0, s}(n)=B_{n+s}$, the generating function $\sum_{n=0}^{\infty} B_{n+s} \frac{x^{n}}{n !}$ equals $e^{e^{x}-1}$ times a polynomial of exact degree $s$. From this, for all $s, r$ the space of all polynomials in $e^{x}$ of degree at most $s+r$ times $e^{e^{x}-1}$ is spanned by the set of generating functions $\sum_{n=0}^{\infty} B_{n+m} \frac{x^{n}}{n !}$ as $m$ runs over all integers $0,1, \ldots, s+r$. Since the generating function for $g_{r, s}(n)$ lies in this span,

$$
\sum_{n=0}^{\infty} g_{r, s}(n) \frac{x^{n}}{n !}=\sum_{m=0}^{s+r} \beta_{s, r, m} \sum_{n=0}^{\infty} B_{n+m} \frac{x^{n}}{n !}
$$

for some rational numbers $\beta_{s, r, m}$. It follows that for all $n$,

$$
g_{r, s}(n)=\sum_{m=0}^{s+r} \beta_{s, r, m} B_{n+m}
$$

For a sequence, $\mathbf{r}=\left\{r_{0}, r_{1}, \cdots, r_{k}\right\}$, of rational numbers and a polynomial $Q \in$ $\mathbb{Q}\left[y_{1}, \cdots, y_{k}, m\right]$ define

$$
M(k, Q, \mathbf{r}, n, x):=\sum_{1 \leq x_{1}<x_{2}<\ldots<x_{k} \leq n} Q\left(x_{1}, \ldots, x_{k}, n\right) \prod_{i=0}^{k}\left(x+r_{i}\right)^{x_{i+1}-x_{i}-1},
$$

where $x_{0}=0, x_{k+1}=n+1$.

Lemma 7. Fix $k$, let $Q \in \mathbb{Z}\left[y_{1}, \cdots, y_{k}, m\right]$ and $\boldsymbol{r}=\left\{r_{0}, r_{1}, \cdots, r_{k}\right\}$ be a sequence of rational numbers. As defined above, $M(k, Q, r, n, x)$ is a rational linear combination of terms of the form

$$
F(n) G(x)\left(x+r_{i}\right)^{n-k},
$$

where $F \in \mathbb{Q}[n], G \in \mathbb{Q}[x]$ are polynomials.

Proof. The proof is by induction on $k$. If $k=0$ then definitionally, $M(k, Q, \mathbf{r}, n, x)=$ $Q(n)\left(x+r_{0}\right)^{n}$, providing a base case for our result. Assume that the lemma holds for $k$ one smaller. For this, fix the values of $x_{1}, \ldots, x_{k-1}$ in the sum and consider the resulting sum over $x_{k}$. Then

$$
\begin{aligned}
& M(k, Q, \mathbf{r}, n, x) \\
& =\sum_{1 \leq x_{1}<x_{2}<\ldots<x_{k-1} \leq n-1} \prod_{i=0}^{k-2}\left(x+r_{i}\right)^{x_{i+1}-x_{i}-1} \\
& \quad \times \sum_{x_{k-1}<x_{k} \leq n} Q\left(x_{1}, \ldots, x_{k}, n\right)\left(x+r_{k-1}\right)^{x_{k}-x_{k-1}-1}\left(x+r_{k}\right)^{n-x_{k}} .
\end{aligned}
$$

Consider the inner sum over $x_{k}$ : 
If $r_{k-1}=r_{k}$, then the product of the last two terms is always $\left(x+r_{k}\right)^{n-x_{k-1}-2}$, and thus the sum is some polynomial in $x_{1}, \ldots, x_{k-1}, n$ times $\left(x+r_{k}\right)^{n-x_{k-1}-2}$. The remaining sum over $x_{1}, \ldots, x_{k-1}$ is exactly of the form $M\left(k-1, Q^{\prime}, \mathbf{r}^{\prime}, n-1, x\right)$, for some polynomial $Q^{\prime}$, and thus, by the inductive hypothesis, of the correct form.

If $r_{k-1} \neq r_{k}$, the sum is over pairs of non-negative integers $a=x_{k}-x_{k-1}-1$ and $b=n-x_{k}-1$ summing to $n-x_{k-1}-2$ of some polynomial, $Q^{\prime}$ in $a$ and $n$ and the other $x_{i}$ times $\left(x+r_{k-1}\right)^{a}\left(x+r_{k}\right)^{b}$. Letting $y=\left(x+r_{k-1}\right)$ and $z=\left(x+r_{k}\right)$, this is a sum of $Q^{\prime}\left(x_{i}, n, a\right) y^{a} z^{b}$. Let $d$ be the $a$ degree of $Q^{\prime}$. Multiplying this sum by $(y-z)^{d+1}$, yields, by standard results, a polynomial in $y$ and $z$ of degree $n-x_{k-1}-2+(d+1)$ in which all terms have either $y$ exponent or $z$ exponent at least $n-x_{k-1}-1$. Thus, this inner sum over $x_{k}$ when multiplied by the non-zero constant $\left(r_{k-1}-r_{k}\right)^{d+1}$ yields the sum of a polynomial in $x, n, x_{1}, \ldots, x_{k-1}$ times $\left(x+r_{k-1}\right)^{n-x_{k-1}-2}$ plus another such polynomial times $\left(x+r_{k-1}\right)^{n-x_{k-1}-2}$. Thus, $M(k, Q, \mathbf{r}, n, x)$ can be written as a linear combination of terms of the form $G(x) M\left(k-1, Q^{\prime}, \mathbf{r}^{\prime}, n, x\right)$. The inductive hypothesis is now enough to complete the proof.

Turn next to the proof of Theorem 2. Proof of Theorem 2

It suffices to prove this Theorem for simple statistics. Thus, it suffices to prove that for any pattern $P$ and polynomial $Q$ that

$$
M\left(f_{P, Q} ; n\right)=\sum_{\lambda \in \Pi_{n}} f_{P, Q}(\lambda)=\sum_{\lambda \in \Pi_{n}} \sum_{s \in P \lambda} Q(s)
$$

is given by a shifted Bell polynomial in $n$. As a first step, interchange the order of summation over $s$ and $\lambda$ above. Hence,

$$
M\left(f_{P, Q} ; n\right)=\sum_{s \in[n]^{k}} Q(s) \sum_{\substack{\lambda \in \Pi(n) \\ s \in P \lambda}} 1 .
$$

To deal with the sum over $\lambda$ above, first consider only the blocks of $\lambda$ that contain some element of $s$. Equivalently, let $\lambda^{\prime}$ be obtained from $\lambda$ by replacing all of the blocks of $\lambda$ that are disjoint from $s$ by their union. To clarify this notation, let $\Pi^{\prime}(n)$ denote the set of all set partitions of $[n]$ with at most one marked block. For $\lambda^{\prime} \in \Pi^{\prime}(n)$, say that $s \in_{P} \lambda^{\prime}$ if $s$ in an occurrence of $P$ in $\lambda^{\prime}$ as a regular set partition so that additionally the non-marked blocks of $\lambda^{\prime}$ are exactly the blocks of $\lambda^{\prime}$ that contain some element of $s$. For $\lambda^{\prime} \in \Pi^{\prime}(n)$ and $\lambda \in \Pi(n)$, say that $\lambda$ is a refinement of $\lambda^{\prime}$ if the unmarked blocks in $\lambda^{\prime}$ are all parts in $\lambda$, or equivalently, if $\lambda$ can be obtained from $\lambda^{\prime}$ by further partitioning the marked block. Denote $\lambda$ being a refinement of $\lambda^{\prime}$ as $\lambda \vdash \lambda^{\prime}$. Thus, in the previous computation of $M\left(f_{P, Q} ; n\right)$, letting $\lambda^{\prime}$ be the marked partition obtained by replacing the blocks in $\lambda$ disjoint from $s$ by their union:

$$
M\left(f_{P, Q} ; n\right)=\sum_{s \in[n]^{k}} Q(s) \sum_{\substack{\lambda^{\prime} \in \Pi^{\prime}(n) \\ s \in P \lambda^{\prime}}} \sum_{\substack{\lambda \in \Pi(n) \\ \lambda \vdash \lambda^{\prime}}} 1 .
$$

Note that the $\lambda$ in the final sum above correspond exactly to the set partitions of the marked block of $\lambda^{\prime}$. For $\lambda^{\prime} \in \Pi^{\prime}(n)$, let $\left|\lambda^{\prime}\right|$ be the size of the marked block of $\lambda^{\prime}$. Thus,

$$
M\left(f_{P, Q} ; n\right)=\sum_{s \in[n]^{k}} Q(s) \sum_{\substack{\lambda^{\prime} \in \Pi^{\prime}(n) \\ s \in P \lambda^{\prime}}} B_{\left|\lambda^{\prime}\right|} .
$$


Remark 13. This is valid even when the marked block is empty.

Dealing directly with the Bell numbers above will prove challenging, so instead compute the generating function

$$
M(P, Q, n, x):=\sum_{s \in[n]^{k}} Q(s) \sum_{\substack{\lambda^{\prime} \in \Pi^{\prime}(n) \\ s \in P \lambda^{\prime}}} x^{\left|\lambda^{\prime}\right|} .
$$

After computing this, extract the coefficients of $M(P, Q, n, x)$ and multiply them by the appropriate Bell numbers.

To compute $M(P, Q, n, x)$, begin by computing the value of the inner sum in terms of $s=\left(x_{1}<x_{2}<\ldots<x_{k}\right)$ that preserves the consecutivity relations of $P$ (namely those in $\mathbf{C}(P))$. Denote the equivalence classes in $P$ by $1,2, \ldots, \ell$. Let $z_{i}$ be a representative of this $i$ th equivalence class. Then, an element $\lambda^{\prime} \in \Pi^{\prime}(n)$ so that $s \in_{P} \lambda^{\prime}$ can be thought of as a set partition of $[n]$ into labeled equivalence classes $0,1, \ldots, \ell$, where the 0 th class is the marked block, and the $i$ th class is the block containing $x_{z_{i}}$. Thus, think of the set of such $\lambda^{\prime}$ as the set of maps $g:[n] \rightarrow\{0,1, \ldots, \ell\}$ so that:

1. $g\left(x_{j}\right)=i$ if $j$ is in the $i$ th equivalence class

2. $g(x) \neq i$ if $x<x_{j}, j \in \mathbf{F}(P)$ and $j$ is in the $i$ th equivalence class

3. $g(x) \neq i$ if $x>x_{j}, j \in \mathbf{L}(P)$ and $j$ is in the $i$ th equivalence class

4. $g(x) \neq i$ if $x_{j}<x<x_{j^{\prime}},\left(j, j^{\prime}\right) \in \mathbf{A}(P)$ and $j, j^{\prime}$ are in the $i$ th equivalence class

It is possible that no such $g$ will exist if one of the latter three properties must be violated by some $x=x_{h}$. If this is the case, this is a property of the pattern $P$, and not the occurrence $s$, and thus, $M\left(f_{P, Q} ; n\right)=0$ for all $n$. Otherwise, in order to specify $g$, assign the given values to $g\left(x_{i}\right)$ and each other $g(x)$ may be independently assigned values from the set of possibilities that does not violate any of the other properties. It should be noted that 0 is always in this set, and that furthermore, this set depends only which of the $x_{i}$ our given $x$ is between. Thus, there are some sets $S_{0}, S_{1}, \ldots, S_{k} \subseteq\{0,1, \ldots, \ell\}$, depending only on $s$, so that $g$ is determined by picking functions

$$
\left\{1, \ldots, x_{1}-1\right\} \rightarrow S_{0},\left\{x_{1}+1, \ldots, x_{2}-1\right\} \rightarrow S_{1}, \ldots,\left\{x_{k}+1, \ldots, n\right\} \rightarrow S_{k}
$$

Thus, the sum over such $\lambda^{\prime}$ of $x^{\left|\lambda^{\prime}\right|}$ is easily seen to be

$$
\left(x+r_{0}\right)^{x_{1}-1}\left(x+r_{i}\right)^{x_{2}-x_{1}-1} \cdots\left(x+r_{k-1}\right)^{x_{k}-x_{k-1}-1}\left(x+r_{k}\right)^{n-x_{k}},
$$

where $r_{i}=\left|S_{i}\right|-1$ (recall $\left|S_{i}\right|>0$, because $0 \in S_{i}$ ). For such a sequence, $\mathbf{r}$ of rational numbers define

$$
M(k, Q, \mathbf{r}, n, x, \mathbf{C}(P)):=\sum_{\substack{1 \leq x_{1}<x_{2}<\ldots<x_{k} \leq n \\\left|x_{i}-x_{j}\right|=1 \text { for }(i, j) \in \mathbf{C}(P)}} Q\left(x_{1}, \ldots, x_{k}, n\right) \prod_{i=0}^{k}\left(x+r_{i}\right)^{x_{i+1}-x_{i}-1},
$$

where, as in Lemma 7, using the notation $x_{0}=0, x_{k+1}=n+1$.

Note that the sum is empty if $\mathbf{C}(P)$ contains nonconsecutive elements. We will henceforth assume that this is not the case. We call $j$ a follower if either $(j-1, j)$ or $(j, j-1)$ are in $\mathbf{C}(P)$. Clearly, the values of all $x_{i}$ are determined only by those $x_{i}$ where $i$ is not a follower. Furthermore, $Q$ is a polynomial in these values and $n$. If $j$ is the index of the 
$i$ th non-follower then let $y_{i}=x_{j}-j+i$. Now, sequences of $x_{i}$ satisfying the necessary conditions correspond exactly to those sequences with $1 \leq y_{1}<y_{2}<\cdots<y_{k-f} \leq n-f$ where $f$ is the total number of followers. Thus,

$$
\begin{aligned}
M(k, Q, \mathbf{r}, n, x, \mathbf{C}(P)) & =\sum_{1 \leq y_{1}<y_{2}<\ldots<y_{k-f} \leq n-f} \widetilde{Q}\left(y_{1}, \ldots, y_{k}, n\right) \prod_{i=0}^{k}\left(x+\widetilde{r}_{i}\right)^{y_{i+1}-y_{i}-1} \\
& =M(k-f, \widetilde{Q}, \widetilde{\mathbf{r}}, n-f, x) .
\end{aligned}
$$

where the $\widetilde{r}_{i}$ are modified versions of the $r_{i}$ to account for the change from $\left\{x_{j}\right\}$ to $\left\{y_{i}\right\}$. In particular, if $x_{j}$ is the $(i+1)^{s t}$ non-follower, then $\widetilde{r_{i}}=r_{j-1}$.

By Lemma 7, $M(k-f, \widetilde{Q}, \widetilde{\mathbf{r}}, n-f, x)$ is a linear combination of terms of the form $F(n) G(x)\left(x+r_{i}\right)^{n-k}$ for polynomials $F \in \mathbb{Q}[n]$ and $G \in \mathbb{Q}[x]$.

Thus, $M\left(f_{P, Q} ; n\right)$ can be written as a linear combination of terms of the form $g_{r, d, \ell, s}(n)$ where $\ell$ is the number of equivalence classes in $P$ and $r, d, s$ are non-negative integers. Therefore, by Lemma $6 M\left(f_{P, Q} ; n\right)$ is a shifted Bell polynomial.

The bound for the upper shift index follows from the fact that $M\left(f_{P, Q} ; n\right)=O\left(n^{N} B_{n}\right)$ and by (13) each term $n^{\alpha} B_{n+\beta}$ is of an asymptotically distinct size. To complete the proof of the result it is sufficient to bound the lower shift index of the Bell polynomial. By (15) it is clear the largest power of $x$ in each term is $(n-k)$. Thus, from Lemma 6 , the resulting shift Bell polynomials can be written with minimum lower shift index $-k$. This completes the proof.

Next turn to the proof of Theorem 1. To this end, introduce some notation.

Definition 3. Given three patterns $P_{1}, P_{2}, P_{3}$, of lengths $k_{1}, k_{2}, k_{3}$, say that a merge of $P_{1}$ and $P_{2}$ onto $P_{3}$ is a pair of strictly increasing functions $m_{1}:\left[k_{1}\right] \rightarrow\left[k_{3}\right], m_{2}:\left[k_{2}\right] \rightarrow\left[k_{3}\right]$ so that

1. $m_{1}\left(\left[k_{1}\right]\right) \cup m_{2}\left(\left[k_{2}\right]\right)=\left[k_{3}\right]$

2. $m_{1}(i) \sim_{P_{3}} m_{1}(j)$ if and only if $i \sim_{P_{1}} j$, and $m_{2}(i) \sim_{P_{3}} m_{2}(j)$ if and only if $i \sim_{P_{2}} j$

3. $i \in \mathbf{F}\left(P_{3}\right)$ if and only if there exists either a $j \in \mathbf{F}\left(P_{1}\right)$ so that $i=m_{1}(j)$ or a $j \in \mathbf{F}\left(P_{2}\right)$ so that $i=m_{2}(j)$

4. $i \in \mathbf{L}\left(P_{3}\right)$ if and only if there exists either a $j \in \mathbf{L}\left(P_{1}\right)$ so that $i=m_{1}(j)$ or a $j \in \mathbf{L}\left(P_{2}\right)$ so that $i=m_{2}(j)$

5. $\left(i, i^{\prime}\right) \in \mathbf{A}\left(P_{3}\right)$ if and only if there exists either a $\left(j, j^{\prime}\right) \in \mathbf{A}\left(P_{1}\right)$ so that $i=m_{1}(j)$ and $i^{\prime}=m_{1}\left(j^{\prime}\right)$ or a $\left(j, j^{\prime}\right) \in \mathbf{A}\left(P_{2}\right)$ so that $i=m_{2}(j)$ and $i^{\prime}=m_{2}\left(j^{\prime}\right)$

6. $\left(i, i^{\prime}\right) \in \mathbf{C}\left(P_{3}\right)$ if and only if there exists either a $\left(j, j^{\prime}\right) \in \mathbf{C}\left(P_{1}\right)$ so that $i=m_{1}(j)$ and $i^{\prime}=m_{1}\left(j^{\prime}\right)$ or a $\left(j, j^{\prime}\right) \in \mathbf{C}\left(P_{2}\right)$ so that $i=m_{2}(j)$ and $i^{\prime}=m_{2}\left(j^{\prime}\right)$

Such a merge is denoted as $m_{1}, m_{2}: P_{1}, P_{2} \rightarrow P_{3}$.

Note that the last four properties above imply that given $P_{1}$ and $P_{2}$, a merge (including a pattern $P_{3}$ ) is uniquely defined by maps $m_{1}, m_{2}$ and an equivalence relation $\sim_{P_{3}}$ satisfying (1) and (2) above.

Lemma 8. Let $P_{1}$ and $P_{2}$ be patterns. For any $\lambda$ there is a one-to-one correspondence:

$$
\left\{\left(s_{1}, s_{2}\right): s_{1} \in_{P_{1}} \lambda, s_{2} \in P_{2} \lambda\right\} \leftrightarrow\left\{P_{3}, s_{3} \in P_{3} \lambda, a n d m_{1}, m_{2}: P_{1}, P_{2} \rightarrow P_{3}\right\} .
$$


Moreover, under this correspondence

$$
\begin{aligned}
& Q_{m_{1}, m_{2}, Q_{1}, Q_{2}}\left(s_{3}\right):=Q_{1}\left(z_{m_{1}(1)}, z_{m_{1}(2)}, \ldots, z_{m_{1}\left(k_{1}\right)}, n\right) Q_{2}\left(z_{m_{2}(1)}, z_{m_{2}(2)}, \ldots, z_{m_{2}\left(k_{2}\right)}, n\right) \\
& \quad=Q_{1}\left(s_{1}\right) Q_{2}\left(s_{2}\right) .
\end{aligned}
$$

Proof. Begin by demonstrating the bijection defined by Eq. 16 . On the one hand, given $s_{3} \in_{P_{3}} \lambda$ given by $z_{1}<z_{2}<\ldots<z_{k_{3}}$ and $m_{1}, m_{2}: P_{1}, P_{2} \rightarrow P_{3}$, define $s_{1}$ and $s_{2}$ by the sequences $z_{m_{1}(1)}<z_{m_{1}(2)}<\ldots<z_{m_{1}\left(k_{1}\right)}$ and $z_{m_{2}(1)}<z_{m_{2}(2)}<\ldots<z_{m_{2}\left(k_{2}\right)}$. It is easy to verify that these are occurrences of the patterns $P_{1}$ and $P_{2}$ and furthermore that Eq. 17 holds for this mapping.

This mapping has a unique inverse: Given $s_{1}$ and $s_{2}$, note that $s_{3}$ must equal the union $s_{1} \cup s_{2}$. Furthermore, the maps $m_{a}$, for $a=1,2$, must be given by the unique function so that $m_{a}(i)=j$ if and only if the $i^{t h}$ smallest element of $s_{a}$ equals the $j^{t h}$ smallest element of $s_{3}$. Note that the union of these images must be all of [ $\left.k_{3}\right]$. In order for $s_{3}$ to be an occurrence of $P_{3}$ the equivalence relation $\sim_{P_{3}}$ must be that $i \sim_{P_{3}} j$ if and only if the $i^{\text {th }}$ and $j^{t h}$ elements of $s_{3}$ are equivalent under $\lambda$. Note that since $S_{1}$ and $S_{2}$ were occurrences of $P_{1}$ and $P_{2}$, that this must satisfy condition (2) for a merge. The rest of the data associated to $P_{3}$ (namely $\mathbf{F}\left(P_{3}\right), \mathbf{L}\left(P_{3}\right), \mathbf{A}\left(P_{3}\right)$, and $\mathbf{C}\left(P_{3}\right)$ ) is now uniquely determined by $m_{1}, m_{2}, P_{1}, P_{2}$ and the fact that $P_{3}$ is a merge of $P_{1}$ and $P_{2}$ under these maps. To show that $s_{3}$ is an occurrence of $P_{3}$ first note that by construction the equivalence relations induced by $\lambda$ and $P_{3}$ agree. If $i \in \mathbf{F}\left(P_{3}\right)$, then there is a $j \in \mathbf{F}\left(P_{a}\right)$ with $i=m_{a}(j)$ for some $a, j$. Since $s_{a}$ is an occurrence of $P_{a}$, this means that the $j^{\text {th }}$ smallest element of $s_{a}$ in in $\operatorname{First}(\lambda)$. On the other hand, by the construction of $m_{a}$, this element is exactly $z_{m_{a}(j)}=z_{i}$. This if $i \in \mathbf{F}\left(P_{3}\right)$, $z_{i} \in \operatorname{First}(\lambda)$. The remaining properties necessary to verify that $S_{3}$ is an occurrence of $P_{3}$ follow similarly. Thus, having shown that the above map has a unique inverse, the proof of the lemma is complete.

Recall, the number of singleton blocks is denoted $X_{1}$ and it is a simple statistic. To illustrate this lemma return to the example of $X_{1}^{2}$ discussed prior to the lemma. Let $P_{1}=$ $P_{2}$ be the pattern of length 1 with $\mathbf{A}\left(P_{1}\right)=\phi, \mathbf{F}\left(P_{1}\right)=\mathbf{L}\left(P_{1}\right)=1$. Then there are five possible merges of $P_{1}$ and $P_{2}$ into some pattern $P_{3}$. The first choice of $P_{3}$ is $P_{1}$ itself. In which case $m_{1}(1)=m_{2}(1)=1$. The latter choices of $P_{3}$ is the pattern of length 2 with $\mathbf{F}\left(P_{3}\right)=\mathbf{L}\left(P_{3}\right)=\{1,2\}, \mathbf{A}\left(P_{3}\right)=\emptyset$. The equivalence relation on $P_{3}$ could be either the trivial one or the one that relates 1 and 2 (though in the latter case the pattern $P_{3}$ will never have any occurrences in any set partition). In either of these cases, there is a merge with $m_{1}(1)=1$ and $m_{2}(1)=2$ and a second merge with $m_{1}(1)=2$ and $m_{2}(1)=1$. As a result,

$$
\begin{aligned}
& M\left(X_{1}^{2} ; n\right)=\sum_{\lambda \in \Pi(n)} X_{1}(\lambda)^{2}=\sum_{\lambda \in \Pi(n)}\left(\sum_{\substack{x_{1} \\
x_{1} \in \operatorname{First}(\lambda) \\
x_{1} \in \operatorname{Last}(\lambda)}} 1\right)^{2}
\end{aligned}
$$

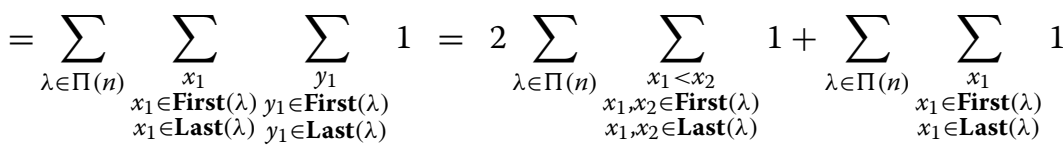


Proof of Theorem 1 The fact that statistics are closed under pointwise addition and scaling follows immediately from the definition. Similarly, the desired degree bounds for these operations also follow easily. Thus only closure and degree bounds for multiplication must be proved. Since every statistic may be written as a linear combination of simple statistics of no greater degree, and since statistics are closed under linear combination, it suffices to prove this theorem for a product of two simple statistics. Thus let $f_{i}$ be the simple statistic defined by a pattern $P_{i}$ of size $k_{i}$ and a polynomial $Q_{i}$. It must be shown that $f_{1}(\lambda) f_{2}(\lambda)$ is given by a statistic of degree at most $k_{1}+k_{2}+\operatorname{deg}\left(Q_{1}\right)+\operatorname{deg}\left(Q_{2}\right)$.

For any $\lambda$

$$
f_{1}(\lambda) f_{2}(\lambda)=\sum_{s_{1} \in P_{1} \lambda, s_{2} \in P_{2} \lambda} Q_{1}\left(s_{1}\right) Q_{2}\left(s_{2}\right) .
$$

Simplify this equation using Lemma 8 , writing this as a sum over occurrences of only a single pattern in $\lambda$.

Applying Lemma 8,

$$
\begin{aligned}
f_{1}(\lambda) f_{2}(\lambda) & =\sum_{s_{1} \in P_{1} \lambda, s_{2} \in P_{2} \lambda} Q_{1}\left(s_{1}\right) Q_{2}\left(s_{2}\right) \\
& =\sum_{P_{3}} \sum_{m_{1}, m_{2}: P_{1}, P_{2} \rightarrow P_{3}} \sum_{s_{3} \in P_{3} \lambda} Q_{m_{1}, m_{2}, Q_{1}, Q_{2}}\left(s_{3}\right), \\
& =\sum_{m_{1}, m_{2}: P_{1}, P_{2} \rightarrow P_{3}} f_{P_{3}, Q_{m_{1}, m_{2}, Q_{1}, Q_{2}}}(\lambda) .
\end{aligned}
$$

Thus, the product of $f_{1}$ and $f_{2}$ is a sum of simple characters. Note that the quantity is a polynomial of $s_{3}$ which is denoted $Q_{m_{1}, m_{2}, Q_{1}, Q_{2}}\left(s_{3}\right)$. Finally, each pattern $P_{3}$ has size at most $k_{1}+k_{2}$ and each polynomial $Q_{m_{1}, m_{2}, Q_{1}, Q_{2}}$ has degree at most $\operatorname{deg}\left(Q_{1}\right)+\operatorname{deg}\left(Q_{2}\right)$. Thus the degree of the product is at most the sum of the degrees.

\section{More data}

This section contains some data for the dimension and intertwining exponent statistics. The moment formulae of Theorem 3 for $k \leq 22$ and the moment formulae for the intertwining exponent for $k \leq 12$ have been computed and are available at [55]. Moreover, the values $f(n, 0, B)$ for $n \leq 238$ and $f_{(i)}(n, 0, B)$ for $n \leq 146$ are available. These sequences can also be found on Sloane's Online Encyclopedia of integer sequences [62].

The remainder of this section contains a small amount of data and observations regarding the distributions $f(n, 0, B)$ and $f_{(i)}(n, 0, B)$ and regarding the shifted Bell polynomials of Theorems 3 and 5 .

\section{Dimension index}

A couple of easy observations: it is clear that

$$
f(n, 0,0)=2^{n-1} .
$$

That is the number of set partitions of [ $n]$ with dimension exponent 0 is $2^{n-1}$. Set partitions of $[n]$ that have dimension exponent 0 must have $n$ appearing in a singleton set or it must appear in a set with $n-1$, thus the result is obtained by recursion. Additionally, the number of set partitions of [ $n]$ with dimension exponent equal to 1 is $n 2^{n-1}$, that is

$$
f(n, 0,1)=n 2^{n-1} .
$$


Curiously, the numbers $f(n, 0, B)$ are smooth (roughly they have many small prime factors), for reasonably sized $B$. This can be established by using the recursion of Theorem 7 . For example,

$$
\begin{aligned}
f(100,0,979)= & 2^{11} \times 3^{7} \times 5^{3} \times 7^{2} \times 11 \times 797 \times 12269 \times 12721 \\
& \times 342966248369 \times 2647544517313 \times 1641377154765701 \\
& \times 16100683847944858147992523687926541327031916811919 \\
f(100,0,2079)= & 2^{27} \times 3^{14} \times 5^{7} \times 7^{4} \times 11^{2} \times 13^{2} \times 17 \times 19 \times 23 \times 29 \times 31 \times 24679914019 \\
& \times 58640283519733 \times 194838881932339884007114639638682100019517
\end{aligned}
$$

Note that $f(100,0,979)$ has 111 digits and $f(100,0,2079)$ has about 100 digits.

In the notation of Theorem 3, these are some values of the first few moments of $d(\lambda)$.

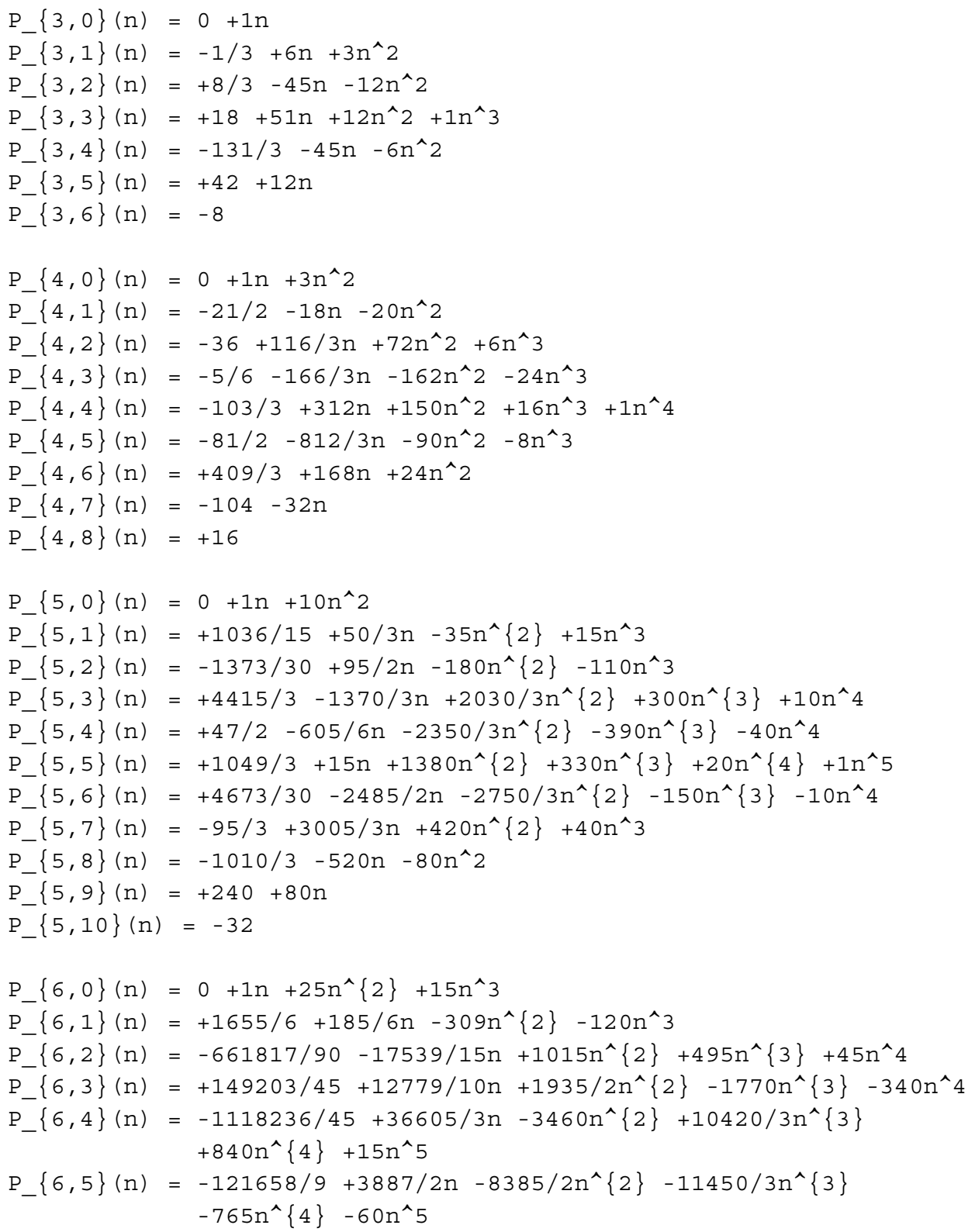




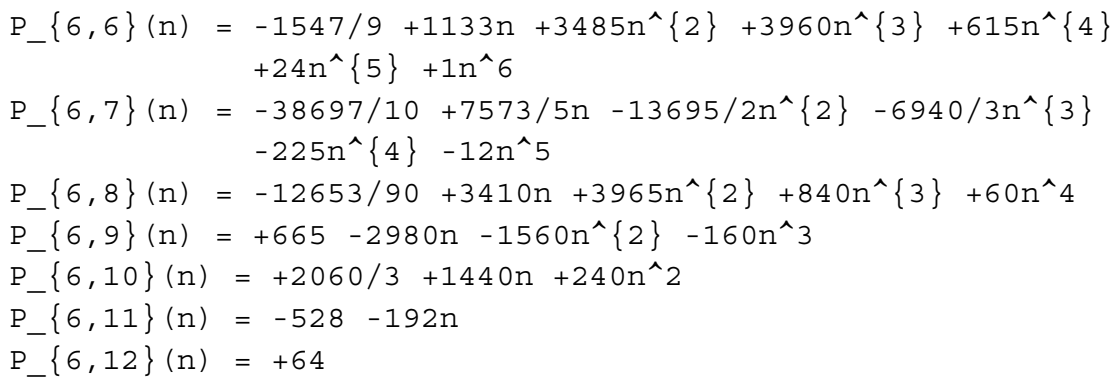

These formulae exhibit a number of properties. Here is a list of some of them.

1. Using the fact that $B_{n+k} \approx n^{k} B_{n}$, each moment $M\left(d^{k} ; n\right)$ has a number of terms with asymptotic of size equal to $n^{2 k} B_{n}$, up to powers of $\log (n)$ (or $\alpha_{n}$ ). Call these terms the leading powers of $n$. The leading 'power' of $n$ contribution is equal to

$$
(n-2 T)^{k} B_{n+k}
$$

where $T$ is the operator given by $T B_{m}=B_{m+1}$. For example, the leading order $n$ contributions for the average is

$$
n B_{n+1}-2 B_{n+2}
$$

and the leading order contribution for the second moment is

$$
n^{2} B_{n+2}-4 n B_{n+3}+4 B_{n+4}
$$

Structure of this sort is necessary because of the asymptotic normality of the dimension exponent (see the forthcoming work [1]). The next remark also concerns this sort of structure.

2. The next order $n$ terms of $M\left(d^{k} ; n\right)$ have size roughly $n^{2 k-1} B_{n}$ and have the shape

$$
\left(\sum_{j \geq 0} C_{j}(-1)^{j+1}\left(\begin{array}{l}
k \\
j
\end{array}\right) n^{k-j} T^{k+j-1}\right) B_{n}
$$

where the constants $C_{j}$ are

$$
C_{j}=2^{j-3}(17-j) j
$$

3. The generating function for the polynomials $P_{0, k}(n)$ seems to be

$$
\sum_{k \geq 0} P_{0, k}(n) \frac{X^{k}}{k !}=\exp \left(\left(e^{X}-1-X\right) n\right) .
$$

We do not have a proof of this observation.

As in the introduction, let $S_{k}(d ; n):=\frac{1}{B_{n}} \sum_{\lambda \in \mathcal{S}_{n}}\left(d(\lambda)-\frac{1}{B_{n}} M(d ; n)\right)^{k}$. From Proposition 2 and the formulae for $M\left(d^{k} ; n\right)$ deduced from Theorem 3 and stated in 
section 'Dimension index' and using SAGE, the asymptotic expansion of the first few $S_{k}$ are as follows:

$$
\begin{aligned}
S_{2}(d ; n)= & \frac{\alpha_{n}^{2}-7 \alpha_{n}+17}{\left(\alpha_{n}+1\right) \alpha_{n}^{3}} n^{3} \\
& +\frac{-8 \alpha_{n}^{7}-29 \alpha_{n}^{6}-136 \alpha_{n}^{5}-207 \alpha_{n}^{4}+69 \alpha_{n}^{3}+407 \alpha_{n}^{2}+116 \alpha_{n}-80}{2 \alpha_{n}^{4}\left(\alpha_{n}+1\right)^{4}} n^{2}+O(n) \\
S_{3}(d ; n)= & \frac{6 \alpha_{n}^{4}-83 \alpha_{n}^{3}+435 \alpha_{n}^{2}-732 \alpha_{n}-881}{3\left(\alpha_{n}+1\right)^{3} \alpha_{n}^{4}} n^{4}+O\left(\frac{n^{3}}{\alpha_{n}^{2}}\right) \\
S_{4}(d ; n)= & 3\left(\frac{\alpha_{n}^{2}-7 \alpha_{n}+17}{\left(\alpha_{n}+1\right) \alpha_{n}^{3}}\right)^{2} n^{6}+O\left(\frac{n^{5}}{\alpha_{n}^{3}}\right) \\
S_{5}(d ; n)= & \frac{10}{3}\left(\frac{\alpha_{n}^{2}-7 \alpha_{n}+17}{\left(\alpha_{n}+1\right) \alpha_{n}^{3}}\right)\left(\frac{6 \alpha_{n}^{4}-83 \alpha_{n}^{3}+435 \alpha_{n}^{2}-732 \alpha_{n}-881}{\left(\alpha_{n}+1\right)^{3} \alpha_{n}^{4}}\right) n^{7}+O\left(\frac{n^{6}}{\alpha_{n}^{4}}\right) \\
S_{6}(d ; n)= & 15\left(\frac{\alpha_{n}^{2}-7 \alpha_{n}+17}{\left(\alpha_{n}+1\right) \alpha_{n}^{3}}\right)^{3} n^{9}+O\left(\frac{n^{8}}{\alpha_{n}^{5}}\right)
\end{aligned}
$$

Remark 14. These asymptotics support the claim that the dimension exponent is normally distributed with mean asymptotic to $\frac{n^{2}}{\log (n)}$ and standard deviation $\sqrt{\frac{n^{3}}{\log (n)^{2}}}$. This result will be established in the forthcoming work [1].

\section{Intertwining index}

Table 2 contains the distribution for of the intertwining exponent for the first few $n$.

In the notation of Theorem 5 , these are some values of the first few moments of $i(\lambda)=c r_{2}(\lambda)$.

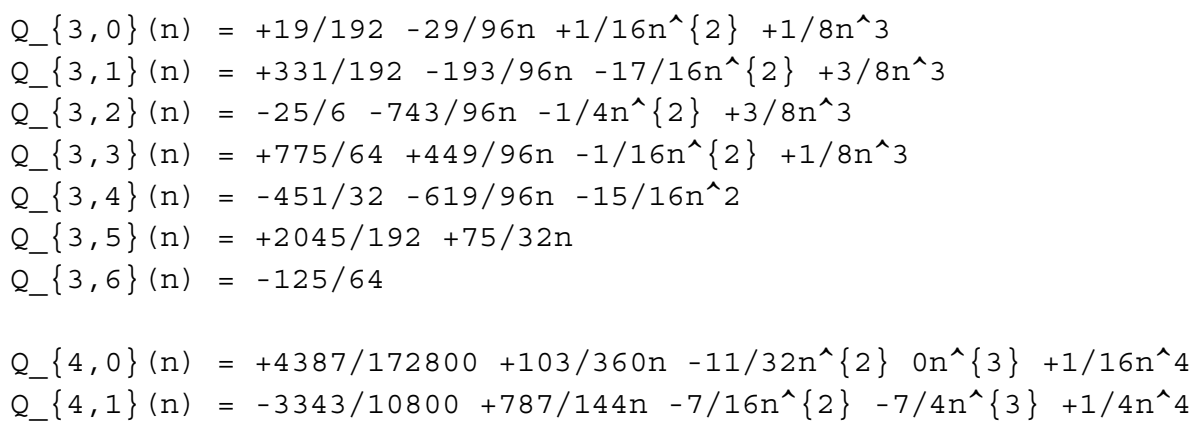

\begin{tabular}{|c|c|c|c|c|c|c|c|c|c|c|c|c|c|}
\hline$n \backslash B$ & 0 & 1 & 2 & 3 & 4 & 5 & 6 & 7 & 8 & 9 & 10 & 11 & 12 \\
\hline 0 & 1 & & & & & & & & & & & & \\
\hline 1 & 1 & & & & & & & & & & & & \\
\hline 2 & 2 & & & & & & & & & & & & \\
\hline 3 & 5 & & & & & & & & & & & & \\
\hline 4 & 14 & 1 & & & & & & & & & & & \\
\hline 5 & 42 & 9 & 1 & & & & & & & & & & \\
\hline 6 & 132 & 55 & 14 & 2 & & & & & & & & & \\
\hline 7 & 429 & 286 & 120 & 35 & 6 & 1 & & & & & & & \\
\hline 8 & 1430 & 1365 & 819 & 364 & 119 & 35 & 7 & 1 & & & & & \\
\hline 9 & 4862 & 6188 & 4900 & 2940 & 1394 & 586 & 203 & 59 & 13 & 2 & & & \\
\hline 10 & 16796 & 27132 & 26928 & 20400 & 12576 & 6846 & 3246 & 1358 & 493 & 153 & 38 & 8 & 1 \\
\hline
\end{tabular}

Table $2 \mathrm{~A}$ table of the distribution of the intertwining exponent $f_{(i)}(n, 0, B)$ 


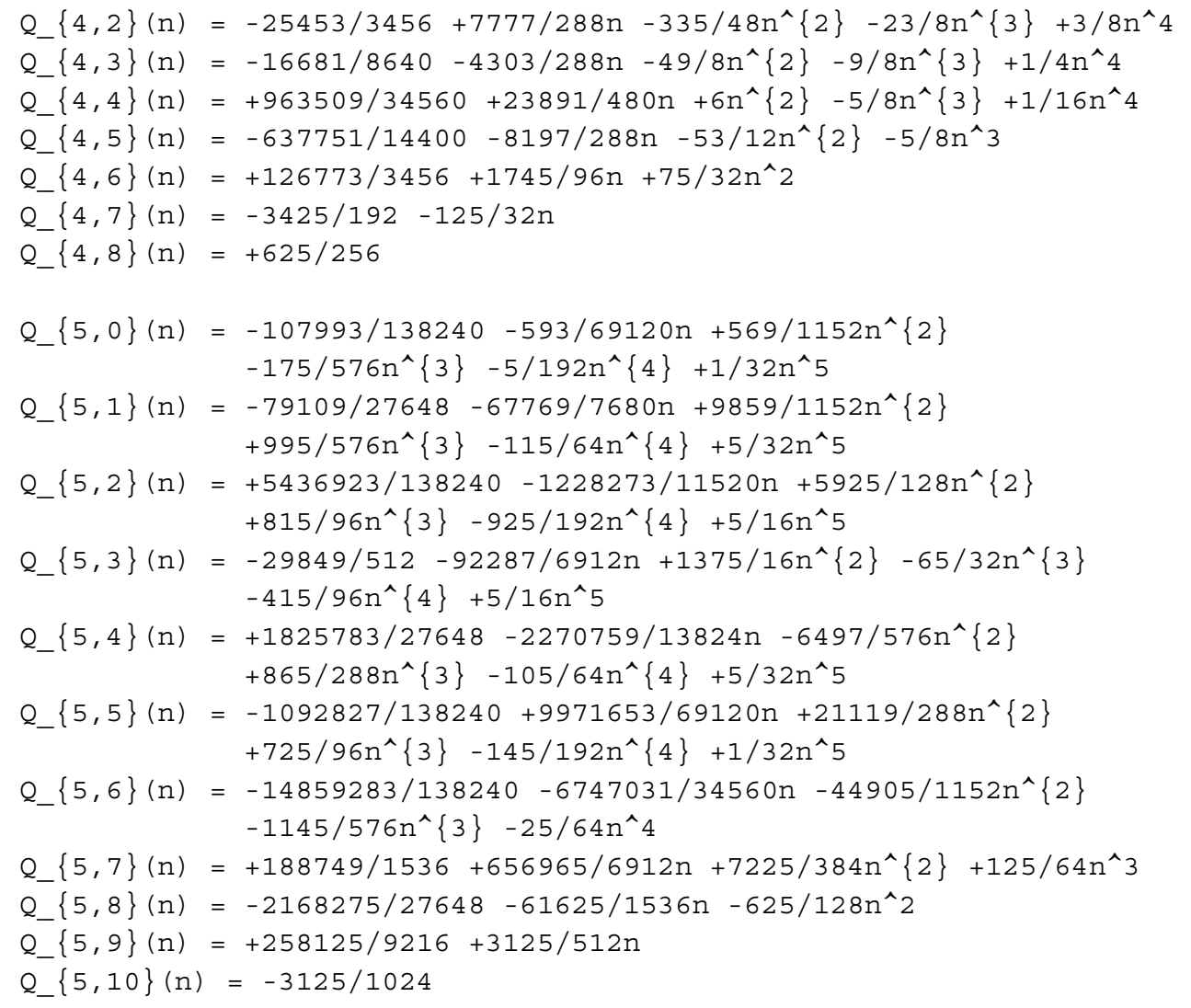

We conjecture that $Q_{j}(n)=0$ for all $j<0$.

The formulae stated previously for $S_{k}(i ; n):=\frac{1}{B_{n}} \sum_{\lambda \in \Pi(n)}\left(i(\lambda)-M(i ; n) / B_{n}\right)^{k}$ with (13) give

$$
\begin{aligned}
S_{2}(i ; n) & =\frac{3 \alpha_{n}^{2}-22 \alpha_{n}+56}{9 \alpha_{n}^{3}\left(\alpha_{n}+1\right)} n^{3} \\
& +\frac{-16 \alpha_{n}^{7}-52 \alpha_{n}^{6}-204 \alpha_{n}^{5}-155 \alpha_{n}^{4}-126 \alpha_{n}^{3}-12 \alpha_{n}^{2}+230 \alpha_{n}+175}{8 \alpha_{n}^{4}\left(\alpha_{n}+1\right)^{4}} n^{2}+O(n) \\
S_{3}(i ; n) & =\frac{\left(\alpha_{n}-5\right)\left(4 \alpha_{n}^{3}-31 \alpha_{n}^{2}+100 \alpha_{n}+99\right)}{8 \alpha_{n}^{4}\left(\alpha_{n}+1\right)^{3}} n^{4}+O\left(\frac{n^{3}}{\alpha_{n}^{3}}\right) \\
S_{4}(i ; n) & =3\left(\frac{\left.3 \alpha_{n}^{2}-22 \alpha_{n}+56\right)^{2}}{9 \alpha_{n}^{3}\left(\alpha_{n}+1\right)}\right)^{2} n^{6}+O\left(\frac{n^{5}}{\alpha_{n}^{3}}\right) \\
S_{5}(i ; n) & =5 \frac{\left(\alpha_{n}-5\right)\left(3 \alpha_{n}^{2}-22 \alpha_{n}+56\right)\left(4 \alpha_{n}^{3}-31 \alpha_{n}^{2}+100 \alpha_{n}+99\right)}{36 \alpha_{n}^{7}\left(\alpha_{n}+1\right)^{4}} n^{7}+O n^{6} \\
S_{6}(i ; n) & =15\left(\frac{\left.3 \alpha_{n}^{2}-22 \alpha_{n}+56\right)^{2}}{9 \alpha_{n}^{3}\left(\alpha_{n}+1\right)}\right) n^{9}+O\left(n^{8}\right)
\end{aligned}
$$

\section{Acknowledgements}

The authors thank the careful referee for a detailed report.

\section{Author details}

${ }^{1}$ Department of Electrical Engineering, Stanford University, Stanford, CA 94305, USA. ${ }^{2}$ Department of Mathematics and Statistics, Sequoia Hall, 390 Serra Mall, Stanford University, Stanford, CA 94305, USA. ${ }^{3}$ Department of Mathematics, Bldg 380, Stanford University, Stanford, CA 94305, USA. ${ }^{4}$ Center for Communications Research, 805 Bunn Dr., Princeton, NJ 08540, USA. 
Received: 17 January 2014 Accepted: 7 February 2014

Published: 17 June 2014

\section{References}

1. Chern, B, Diaconis, P, Kane, DM, Rhoades, RC: Asymptotic normality of set partition statistics associated with supercharacters. in press

2. Chen, WYC, Deng, EYP, Du, RRX, Stanley, RP: Crossings and nestings of matchings and partition. Trans. Amer. Math. Soc. 359(4), 1555-1575 (2007)

3. Stanley, RP: Enumerative Combinatorics. Volume 2. Cambridge Studies in Advanced Mathematics, Vol. 62. Cambridge University Press, Cambridge (1999)

4. Kreweras, G: Sur les partitions noncroisées d'un cycle. Discrete Math. 1, 333-350 (1972)

5. Simion, R: Noncrossing partitions. Discrete Math. 217, 367-409 (2000)

6. Marberg, E: Crossings and nestings in colored set partitions. Electron. J. Combin. 20(4) (2013). Research Paper 6

7. Mansour, T: Combinatorics of Set Partitions. Discrete Mathematics and its Applications. CRC Press, Boca Raton, FL (2013)

8. Shattuck, M: Recounting the number of rises, levels, and descents in finite set partitions. Integers. 10, 179-185 (2010)

9. Kasraoui, A: Average values of some z-parameters in a random set partition. Electron. J. Combinatorics. 18(1), 42 (2011). Paper 228

10. Mansour, T, Shattuck, M: Enumerating finite set partitions according to the number of connectors. Online J. Anal. Combinatorics. 6, 17 (2011). Article 3

11. Knopfmacher, A, Mansour, T, Wagne, S: Records in set partitions. Electron. J. Combinatorics. 17, 14 (2010). Paper 109

12. Graham, RL, Knuth, DE, Patashnik, O: Concrete Mathematics: A Foundation for Computer Science. 2nd edn. Addison-Wesley Publishing Company, Reading, MA (1994)

13. Knuth, D: The Art of Computer Programming vol. 4a: Combinatorial Algorithms. Part I. Addison-Wesley, Upper Saddle River, New Jersey (2011)

14. Stanley, RP: Enumerative Combinatorics. Volume 1, 2nd edn. Cambridge Studies in Advanced Mathematics, Vol. 49. Cambridge University Press, Cambridge (2012)

15. Riordan, J: An Introduction to Combinatory Analysis. Wiley, New York (1958)

16. Pitman, J: Combinatorial Stochastic Processes. Springer, Berlin (2006)

17. Garsia, A: An expose of the Mullin-Rota theory of polynomials of binomial type. Linear Multilinear Algebra. 1,47-65 (1973)

18. Knuth, D: Convolution polynomials. Math. J. 2, 67-78 (1992)

19. Halverson, T, Ram, A: Partition algebras. Eur. J. Combin. 26(6), 869-921 (2005)

20. Aguiar, M, Mahajan, S: Monoidal functors, species and Hopf Algebras. CRM monograph series, Vol. 29. American Mathematical Society, Providence, RI (2010)

21. Kasraoui, A, Zeng, J: Distribution of crossings, nestings and alignments of two edges in matchings and partitions. Electron. J. Combinatorics. 12(1) (2006). Paper 33

22. Kasraoui, A: On the limiting distribution of some numbers of crossings in set partitions. arXiv:1301.6540 [math.CO]

23. Fristedt, B: The structure of random partitions of large sets. Technical report (1987)

24. Hwang, HK: On convergence rates in the central limit theorems for combinatorial structures. Eur. J. Combin. 19(3), 329-343 (1998)

25. Stam, AJ: Generation of random partitions of a set by an urn model. J. Combin. Theory Series A. 35, 231-240 (1983)

26. Pitman, J: Some probabilistic aspects of set partitions. Amer. Math. Mon. 104, 201-209 (1997)

27. Erdös, P, Turán, P: On some problems of statistical group theory, I. Z. Whhr. Verw. Gebiete. 4, 151-163 (1965)

28. Erdös, P, Turán, P: On some problems of statistical group theory, II. Acta Math. Acad. Sci. Hun. 18, 151-163 (1967)

29. Erdös, P, Turán, P: On some problems of statistical group theory, III. Acta Math. Acad. Sci. Hun. 18, 309-320 (1967)

30. Erdös, P, Turán, P: On some problems of statistical group theory, IV. Acta Math. Acad. Sci. Hun. 19, 413-435 (1968)

31. Erdös, P, Turán, P: On some problems of statistical group theory. V. Periodica Math. Hung. 1, 5-13 (1971)

32. Erdös, P, Turán, P: On some problems of statistical group theory, VI. J. Ind. Math. Soc. 34, 175-192 (1970)

33. Erdös, P, Turán, P: On some problems of statistical group theory, VII. Periodica Math. Hung. 2, 149-163 (1972)

34. Fulman, J: Random matrix theory over finite fields. Bull. Amer. Math. Soc. 34, 51-85 (2002)

35. Diaconis, P, Fulman, J, Guralnick, R: On fixed points of random permutations. J. Alg. Comb. 28, 189-218 (2008)

36. Newman, MF: Groups of prime-power order. In: Kov'acs LG (ed.) Groups-Canberra 1989. Lecture Notes in Mathematics vol. 1456, pp. 49-62. Springer, Berlin, (1990)

37. Kerov, S: Asymptotic Representation Theory of the Symmetric Group and Its Applications in Analysis. In: Translations of Mathematical Monographs vol, 219. American Mathematical Society, Providence, Rl, (2003)

38. Turán, P: Remarks on the characters belonging to the irreducible representations of the symmetric group $S_{n}$ of $n$ letters. Fourier analysis and approximation theory (Proc. Colloq., Budapest, 1976, vol. ii. In: Colloq. Math. Soc. János Bolyai, vol. 19, pp. 871-875, Berlin, (1978)

39. Stanley, RP: Increasing and decreasing subsequences and their variants. In: Proceedings of International Congress of Mathematical Society, pp. 545-579, Zurich, (2006)

40. Kerov, S, Vershik, A: Asymptotics of the Plancherel measure of the symmetric group and the limiting form of Young tableaux. Docl. Akad. Nauk. 233, 1024-1027 (1977)

41. Logan, B, Shepp, L: A variational problem for random Young tableaux. Adv. Math. 26, 206-222 (1977)

42. Baik, J, Deift, $P$, Johansson, $K$ : On the distribution of the length of the longest increasing subsequence of random permutations. J. Amer. Math. Soc. 12, 1119-1178 (1999)

43. Anderson, GW, Guionnet, A, Zeitouni, O: Introduction to Random Matrices. Cambridge Press, Cambridge (2009)

44. Forrester, P: Log-gases and Random Matrices. Princeton University Press, Princeton (2010)

45. Arias-Castro, E, Diaconis, P, Stanley, R: A super-class walk on upper-triangular matrices. J. Algebra 278(2), 739-765 (2004)

46. André, C: Basic characters of unitriangular group. J. Algebra 175, 287-319 (1995) 
47. André, C: Irreducible characters of finite algebra groups. In: Santana, AP, Duarte, AL, JFQ (eds.) Matrices and Group Representations. Sér B no. 19, pp. 65-80. Textos Mat, Coimbra, (1998)

48. André, C: Basic characters of the unitriangular group (for arbitrary primes), Vol. 130 (2002)

49. Yan, N: Representation theory of the finite unipotent linear group. PhD thesis, Pennsylvania State University. Department of Mathematics (2001)

50. Aguiar, M, André, C, Benedetti, C, Bergeron, N, Chen, Z, Diaconis, P, Hendrickson, A, Hsiao, S, Isaacs, IM, Jedwab, A Johnson, K, Karaali, G, Lauve, A, Le, T, Lewis, S, Li, H, Magaard, K, Marberg, E, Novelli, J-C, Pang, A, Saliola, F, Tevlin, L, Thibon, JY, Thiem, N, Venkateswaran, V, Vinroot, CR, Yan, N, Zabrocki, M: Supercharacters, symmetric functions in noncommuting variables, and related Hopf algebras. Adv. Math. 229(4), 2310-2337 (2012)

51. Aguiar, $\mathrm{M}$, Bergeron, $\mathrm{N}$, Thiem, $\mathrm{N}$ : Hopf monoids from class functions on unitriangular matrices. Algebra and Number Theory, 7-7, 1743-1779 (2013)

52. Diaconis, P, Isaacs, IM: Supercharacters and superclasses for algebra groups. Trans. Amer. Math. Soc. 360, 2359-2392 (2008)

53. Diaconis, P, Thiem, N: Supercharacter formulas for pattern groups. Trans. Amer. Math. Soc. 361, 3501-3533 (2009)

54. Marberg, E: Actions and identities on set partitions. Electron. J. Combinatorics. 19(1), 31 (2013). Paper 28

55. Rhoades, RC (2013). http://math.stanford.edu/ rhoades/RESEARCH/papers.html

56. Bergeron, $\mathrm{N}$, Thiem, $\mathrm{N}$ : A supercharacter table decomposition via power-sum symmetric functions. Int. J. Algebra Comput. (IJAC). 23-4, 763-778 (2013)

57. Lunnon, WF, Pleasants, PAB, Stephens, NM: Arithmetic properties of Bell numbers to a composite modulus i. Acta Arith. 35(1), 1-16 (1979)

58. Montgomery, PL, Nahm, SJr: SSW: The period of the Bell numbers modulo a prime. Math. Comp. 79(271), 1793-1800 (2010)

59. Nijenhuis, A, Wilf, HS: Combinatorial Algorithms. For Computers and Calculators. 2nd edn. Computer Science and Applied Mathematics. Academic Press, Inc, New York-London (1978)

60. Ruskey F: Combinatorial Object Server (2013). http://www.theory.csc.uvic.ca/ cos/

61. Bruijn, de: NG. Asymptotic Methods in Analysis.Dover, New York (1981)

62. Sloane N: Online Encyclopedia of Integer Sequences (2013). http://oeis.org/

doi:10.1186/2197-9847-1-2

Cite this article as: Chern et al: Closed expressions for averages of set partition statistics. Research in the Mathematical Sciences 2014 1:2.

\section{Submit your manuscript to a SpringerOpen ${ }^{\circ}$ journal and benefit from:}

- Convenient online submission

- Rigorous peer review

- Immediate publication on acceptance

- Open access: articles freely available online

- High visibility within the field

- Retaining the copyright to your article

Submit your next manuscript at $\boldsymbol{\wedge}$ springeropen.com 